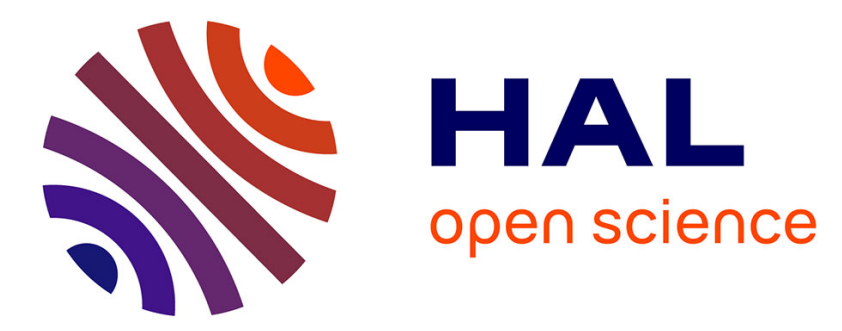

\title{
Gas phase dynamics, conformational transitions and spectroscopy of charged saccharides: the oxocarbenium ion, protonated anhydrogalactose and protonated methyl galactopyranoside
}

\author{
Pierre Carcabal, M. Dvores, P. Maître, J. Simons, R. Gerber
}

\section{To cite this version:}

Pierre Carcabal, M. Dvores, P. Maître, J. Simons, R. Gerber. Gas phase dynamics, conformational transitions and spectroscopy of charged saccharides: the oxocarbenium ion, protonated anhydrogalactose and protonated methyl galactopyranoside. Physical Chemistry Chemical Physics, 2020, 22 (7), pp.4144-4157. 10.1039/c9cp06572e . hal-03000470

\author{
HAL Id: hal-03000470 \\ https://hal.science/hal-03000470
}

Submitted on 15 Nov 2020

HAL is a multi-disciplinary open access archive for the deposit and dissemination of scientific research documents, whether they are published or not. The documents may come from teaching and research institutions in France or abroad, or from public or private research centers.
L'archive ouverte pluridisciplinaire HAL, est destinée au dépôt et à la diffusion de documents scientifiques de niveau recherche, publiés ou non, émanant des établissements d'enseignement et de recherche français ou étrangers, des laboratoires publics ou privés. 

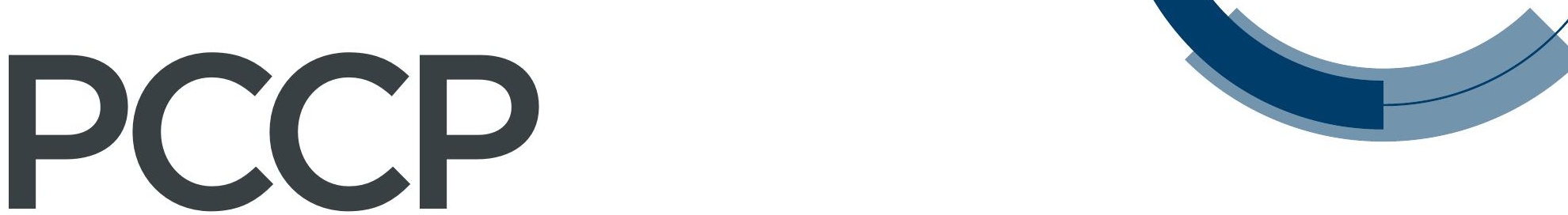

Physical Chemistry Chemical Physics

\section{Accepted Manuscript}

This article can be cited before page numbers have been issued, to do this please use: $M$. Dvores, P. Carcabal, P. Maitre, J. Simons and R. B. Gerber, Phys. Chem. Chem. Phys., 2020, DOI:

\subsection{9/C9CP06572E.}
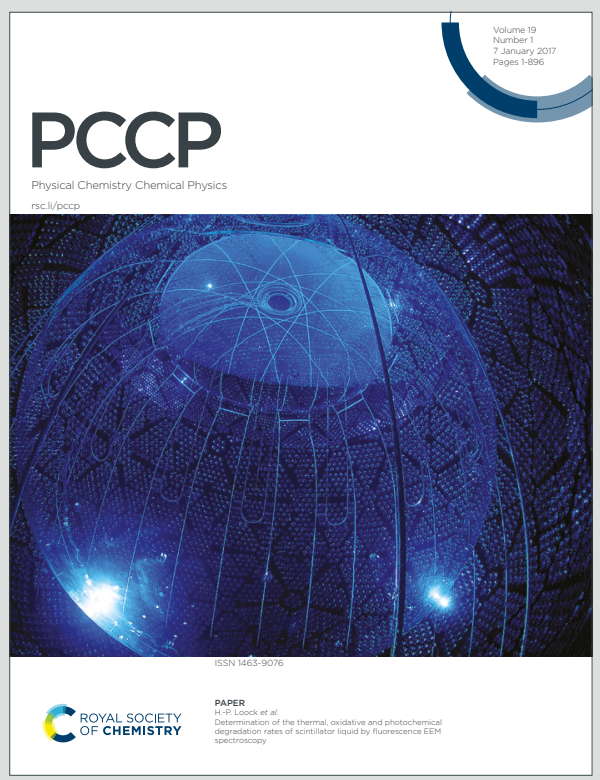

This is an Accepted Manuscript, which has been through the Royal Society of Chemistry peer review process and has been accepted for publication.

Accepted Manuscripts are published online shortly after acceptance, before technical editing, formatting and proof reading. Using this free service, authors can make their results available to the community, in citable form, before we publish the edited article. We will replace this Accepted Manuscript with the edited and formatted Advance Article as soon as it is available.

You can find more information about Accepted Manuscripts in the Information for Authors.

Please note that technical editing may introduce minor changes to the text and/or graphics, which may alter content. The journal's standard Terms \& Conditions and the Ethical guidelines still apply. In no event shall the Royal Society of Chemistry be held responsible for any errors or omissions in this Accepted Manuscript or any consequences arising from the use of any information it contains. 
Received 00th January 20xx, Accepted 00th January 20xx DOI: $10.1039 / \times 0 \times x 00000 x$

\title{
Gas Phase Dynamics, Conformational Transitions and

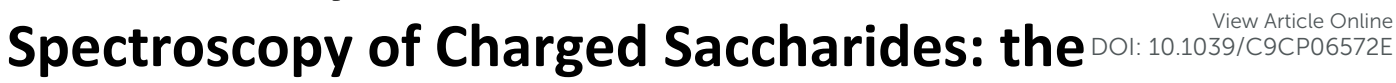 Oxocarbenium Ion, Protonated Anhydrogalactose and Protonated Methyl Galactopyranoside
}

\begin{abstract}
M. P. Dvores, ${ }^{\text {a P. Çarçabal }}{ }^{b}$, P. Maitrec ${ }^{c}$ J.P. Simons ${ }^{d}$ and R. B. Gerber ${ }^{a, e}$
Protonated intermediates are postulated to be involved in the rate determining step of many sugar reactions. This paper presents a study of protonated sugar species, isolated in the gas phase, using a combination of infrared multiple photon dissociation (IRMPD) spectroscopy, classical ab initio molecular dynamics (AIMD) and quantum mechanical vibrational selfconsistent field (VSCF) calculations. It provides a likely identification of the reactive intermediate oxocarbenium ion structure in a D-galactosyl system as well as the saccharide pyrolysis product anhydrogalactose (that suggests oxocarbenium ion stabilization), along with the spectrum of the protonated parent species: methyl D-galactopyranoside- $\mathrm{H}^{+}$. Its vibrational fingerprint indicates intramolecular proton sharing. Classical AIMD simulations for galactosyl oxocarbenium ions, conducted in the temperature range $\sim 300-350 \mathrm{~K}$ (using B3LYP potentials on-the-fly) reveal efficient transitions on the picosecond timescale. Multiple conformers are likely to exist under the experimental conditions and along with static VSCF calculations, they have facilitated the identification of the individual structural motifs of the galactosyl oxocarbenium ion and protonated anhydrogalactose ion conformers that contribute to the observed experimental spectra. These results demonstrate the power of experimental IRMPD spectroscopy combined with dynamics simulations and with computational spectroscopy at the anharmonic level to unravel conformer structures of protonated saccharides, and to provide information on their lifetimes.
\end{abstract}

\section{Introduction}

Saccharides are common glycan structures in biological systems yet their synthetic preparation and manipulation remains a challenge. Currently, a host of methods exists involving typically complex schemes for the activation and protection of the glycosyl donor/acceptor pair to facilitate non-enzymatic oligosaccharide syntheses. 1,2 However, the yield and selectivity are often low ${ }^{3}$ and the realm of feasible products is still quite limited. ${ }^{4-6}$ The regio- and stereoselectivity problems inherent in saccharide reactions are also a source of difficulty in the reverse case: the acid- catalysed hydrolysis of the glyosidic bond. This is a reaction of interest for biomass derived fuel and chemicals production ${ }^{7}$ but side reactions prevent in part the efficient conversion of saccharide biomass to useful materials. ${ }^{8}$ For non-enzymatic reactions of saccharides the current approach, by which each reaction must be treated separately ${ }^{9}$ in order to obtain the desired product, needs to be replaced by a better understanding of the mechanistic principles of saccharide reactions. Namely, how conformation, ${ }^{10}$ ion-pair formation ${ }^{11}$ and glycosyl acceptor nucleophilicity ${ }^{12}$ among other factors steer the selectivity of the reaction. A groundbreaking study characterizing the structures of glycosyl cations and their reduction products with NMR in a superacidic medium has demonstrated the possibility of isolating saccharide reaction intermediates. ${ }^{13}$

a. Institute of Chemistry and the Fritz Haber Research Center, The Hebrew University, Jerusalem 91904, Israel.

b. Insitut des Sciences Moléculaires d'Orsay, ISMO, Univ Paris-Sud; CNRS, bat 210, Univ Paris-Sud 91405 Orsay cedex, France.

c. Laboratoire de Chimie Physique, LCP, Univ Paris-Sud; CNRS, bat 349, Univ Paris-Sud 91405 Orsay cedex, France.

d. Department of Chemistry, Physical and Theoretical Chemistry Laboratory, Oxford University, South Parks Road, Oxford OX1 3QZ, UK.

e. Department of Chemistry, University of California Irvine, CA, 92697, USA.

tElectronic Supplementary Information (ESI) available: [details of any supplementary information available should be included here]. See DOI: $10.1039 / x 0 x x 00000 x$
However, caveats regarding the relevance of these structures in neutral organic solvent ${ }^{14}$ point to the need for other experimental methods to isolate and characterize ionic saccharide reaction intermediates. A gas phase model of non-enzymatic saccharide reactions would provide a key advance towards determining what underlies the outcome of these diastereodifferentiation reactions, offering atomistic level insights into reaction systems that hinge upon the formation of a fleeting oxocarbenium ion intermediate. 11 , $\underline{15-20}$ The gas phase reaction dynamics of oxocarbenium ion formation and decay in model saccharide reaction systems could pinpoint the conformational ${ }^{\ddagger}$-factors that serve to direct the regioand stereoselectivity of the reaction in vacuo. The influence of glycosyl conformation on reactivity, where the oxocarbenium ion is suspected to be a critical intermediate, has previously been the focus of numerous experimental studies in solution:-9, 16, 17, 19, 21-23 for example, acid-catalysed glycosylation, $\underline{21}, \underline{23}$ glycosidation, $\underline{24}, \underline{25}$ hydrolysis $9, \underline{21}, \underline{23}$ and acetolysis ${ }^{22}$ reactions. Enhanced reactivity is thought to be associated with the stabilizing influence these structural changes exert on transition states with significant oxocarbenium ion character. ${ }^{22}$ In a gas phase model, the intrinsic nature of these stabilizing effects can be fully separated from effects that originate or are modified by interactions with solvent.

Protonated saccharides have previously been studied using mass spectrometry combined with infrared multiple photon dissociation (IRMPD) at ambient temperatures, ${ }^{26-28}$ and at low temperature $(0.37 \mathrm{~K})$ with cold-ion infrared spectroscopy in $\mathrm{He}$ droplets. ${ }^{29-32}$ The first report of the characterization of glycosyl cations in the gas phase identified both oxocarbenium and acetoxonium ions among the fragments of fully protected mannosyl cations. ${ }^{26}$ There the mixing of calculated spectra in order to improve the fit to the observed spectrum suggested that more than one isomer derived from acetylated mannuronic acid esters was present under the conditions of the experiment. The enhanced resolution of ring puckering features in the cold-ion infrared 
spectroscopy study of C2 participation in fully protected glycosyl cations has further refined the ability to characterize the defining features of oxocarbenium and acetoxonium ions. ${ }^{31}$ Here too, spectra of glycosyl cations at low temperature indicated coexistence of multiple isomers as well as open-chain configurations. However, the description of the dynamic processes of relevance in these systems such as conformer transitions and proton transfer was limited by the exclusive reliance on single structure methods for spectra calculation.

$A b$ Initio molecular dynamics (AIMD) simulation ${ }^{33}$ has been used extensively to calculate finite temperature anharmonic vibrational spectra while exploring dynamical behaviour under the conditions of the mass spectrometry experiment, thus providing information about the conformational changes which the experimental systems undergo. ${ }^{34}$ Previous molecular dynamics studies of protonated saccharides in the gas phase have examined the vibrational spectroscopy ${ }^{27}$ and dynamics of these species at the picosecond timescale. ${ }^{35}$ The addition of bound solvent to the saccharide systems is possible as well, ${ }^{36-40}$ such that many experimental systems relevant to non-enzymatic saccharide reactions are accessible to simulation.

This study explores the microscopic structure and dynamics of three important protonated saccharide species: the protonated sugar, its oxocarbenium ion fragment and the protonated anhydrosugar. Mass spectrometric techniques and vibrational spectroscopy methods are used to characterize mass selected species, specifically an $\mathrm{m} / \mathrm{z} 195$ fragment corresponding to the protonated sugar and an $m / z 163$ fragment- whose mass could be equivalent to that of an oxocarbenium ion derived from the parent sugar or alternatively, the anhydro-sugar. With only the mass of the experimentally isolated species known, theory is used to interpret the IRMPD spectra and thereby identify the properties of the isolated saccharide ions and their fragments. AIMD simulations, the prime objective of which is to explore transitions between structures as well as to predict populations and lifetimes of conformers, are carried out along with vibrational self-consistent field (VSCF) $\underline{41}, \underline{42}$ calculations to assess the spectroscopy at the anharmonic quantum level. VSCF is a quantum-mechanical method and is often the method of choice for anharmonic frequencies and intensities of polyatomic systems at a well-defined equilibrium structure. Treatment at the anharmonic level must be undertaken in this case as the hydrogenic stretches of the saccharide ions and their oxocarbenium ions are known to involve substantial intrinsic anharmonicities. ${ }^{43}$ These transitions are essential for identification of the experimentally isolated species.

The system presented in this study is the protonated anomeric pair: methyl $\alpha$-D-galactopyranoside $+\mathrm{H}^{+}\left(\alpha \mathrm{MeGal}-\mathrm{H}^{+}\right)$and methyl $\beta$ $D$-galactopyranoside $+\mathrm{H}^{+}\left(\beta \mathrm{MeGal}-\mathrm{H}^{+}\right)$which were generated from an electrospray ionization (ESI) source. These parent ions and their charged fragments were detected with mass spectrometry and probed through IRMPD spectroscopy. For both anomers, an $\mathrm{m} / \mathrm{z}$ 195 species was detected as well as an $m / z 163$ fragment whose mass corresponds to that of an oxocarbenium ion. Protonated $\mathrm{m} / \mathrm{z}$ 163 anhydrogalactoses derived from 1,6 -Anhydro- $\alpha$-Dgalactofuranose and 1,6-Anhydro- $\beta$-D-galactopyranose (levogalactosan) are considered as well in order to determine the contributions to the spectra of non-oxocarbenium ion species that are known products of the acid-catalysed reversion of $\mathrm{P}_{\overline{\mathrm{w}}}$ Article Online

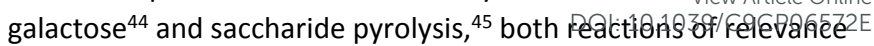
under the experimental conditions, ca. 300-350 K. $.44,45$ Calculations of the IR spectra of the protonated galactopyranoside and anhydrogalactose as well as the galactosyl oxocarbenium ion with AIMD and VSCF, following a low energy conformer search, were used to interpret the experimental spectra. The need for using both of these methods to compute the spectra will be seen later in this study. AIMD simulations of the lowest energy conformer of protonated galactopyranoside, low energy conformers of galactosyl oxocarbenium ion and protonated anhydrogalactose followed the dynamics of these saccharide ions in the estimated experimental temperature range of $\sim 300-350 \mathrm{~K}$.

The structure of the paper is as follows. Section 2 presents the system studied along with the theoretical and experimental methodology. Section 3 presents the findings with analysis and discussion of the comparison of the calculations with the experimental spectra. Concluding remarks are presented in section 4.

\section{System and Methods}

\subsection{Protonated Galactopyranoside Anomers}

This study considers the $\alpha$ - and $\beta$-anomers of methyl Dgalactopyranoside. Specifically, the protonated ions, $\alpha \mathrm{MeGal}-\mathrm{H}^{+}$and $\mathrm{BMeGal} \mathrm{H}^{+}$, are relevant in the IRMPD experiment which isolates the protonated sugars and their fragments. The structures of the protonated species present in the IRMPD experiment are identified with calculations of vibrational spectra. Fig. 1 shows the minimum energy structures of $\alpha \mathrm{MeGal}-\mathrm{H}^{+}, \beta \mathrm{MeGal}-\mathrm{H}^{+}$, galactosyl oxocarbenium ion, protonated 1,6 -Anhydro- $\alpha$-D-galactofuranose and protonated levogalactosan.

\subsection{Computational Methods}

\subsubsection{Low Energy Conformer Search: A low energy conformer} search was conducted at the outset in order to identify conformers of $\alpha \mathrm{MeGal}-\mathrm{H}^{+}, \beta \mathrm{MeGal}-\mathrm{H}^{+}$, galactosyl oxocarbenium ions, protonated 1,6-Anhydro- $\alpha$-D-galactofuranose and protonated levogalactosan that were likely to be present under the experimental conditions. For this purpose, geometric optimizations of $\alpha \mathrm{MeGal}-\mathrm{H}^{+}, \beta \mathrm{MeGal}-\mathrm{H}^{+}$and protonated anhydrogalactoses with initial protonation at $\mathrm{O} 1, \mathrm{O} 2, \mathrm{O} 3, \mathrm{O} 4, \mathrm{O} 5$ and $\mathrm{O} 6$ were performed so as to identify the position of protonation favoured under gas phase conditions for the parent saccharides. Geometric optimization of the galactosyl oxocarbenium ion was performed following the cleavage of the methanol moiety from the protonated galactopyranoside. These optimizations were calculated with Density functional theory using the B3LYP-D $\underline{46}$, 47 functional and a 6$311++G(d, p)$ basis, where all optimizations were performed with the Turbomole 6.3 software package. ${ }^{48}$

The approach used in this study towards finding the structures of the lowest energy conformers of the sugar ion species is based on $a b$ initio optimization of a survey of conformers with reasonable energies. Previous studies of sugars, $, 27, \underline{40}$ peptides, ${ }^{49}$ and G...C base pairs ${ }^{50}$ of comparable system size have employed this approach to successfully identify low energy conformers without taking into account all conformers on the potential energy surface. Evidence 

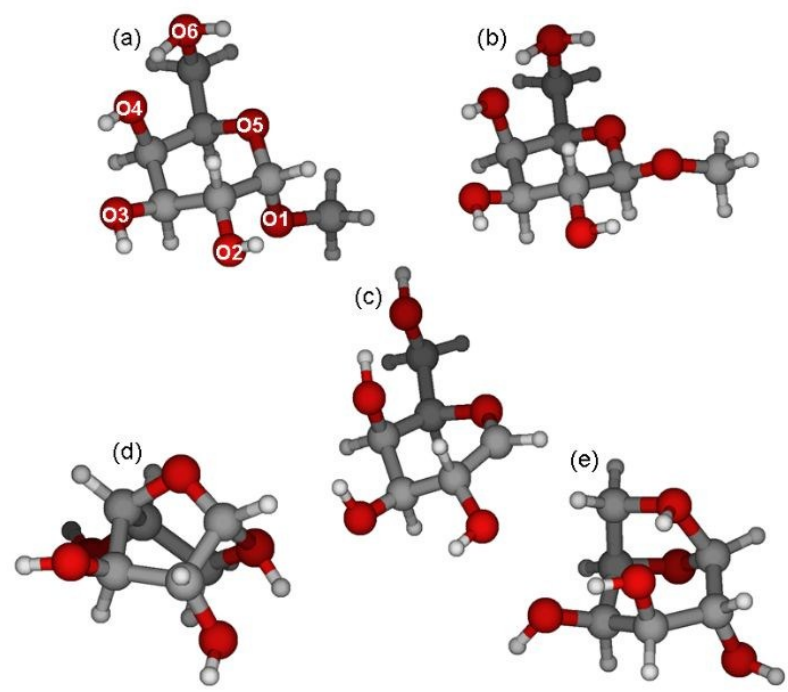

Fig. 1 Minimum energy structures of (a) $\alpha \mathrm{MeGal}-\mathrm{H}^{+}$, (b) $\beta \mathrm{MeGal}-\mathrm{H}^{+}$, (c) galactosyl oxocarbenium ion, (d) protonated 1,6 -Anhydro- $\alpha$-D-galactofuranose and (e) protonated levogalactosan optimized at the B3LYP-D/6-31++G $(d, p)$ level of theory. C atom (grey); $\mathrm{O}$ atom (red); $\mathrm{H}$ atom (white).

for the justification of this limited low energy conformer survey comes from the AIMD simulations, where only truly low energy conformers (within $0.1 \mathrm{eV}$ of the minimum energy structure) had appreciable lifetimes at the $\sim 350 \mathrm{~K}$ simulation temperature (see section 3 ). The use of AIMD simulations as a means of exploring the conformational dynamics is of great importance in this high temperature system. At ambient temperature the gap in energies between the conformers is small enough to assume that isomerization takes place under the experimental conditions, such that a multi-conformer method like AIMD is advantageous. However, it is clear that current approach does not constitute the sole possibility with regards to a conformer survey in this system. Other methods for identifying low energy conformers, particularly recent techniques employing enhanced sampling methods such as metadynamics ${ }^{51}$ or replica exchange dynamics, ${ }^{52}$ could facilitate a further survey of low energy conformers in the present system.

2.2.2 AIMD Simulation: AIMD simulations initiated from the optimized low energy conformers of protonated galactopyranoside, protonated anhydrogalactoses and galactosyl oxocarbenium ions served multiple purposes in this study: to sample the potential energy surface and thereby continue the search for energetically relevant conformers, to study the conformer lifetimes of these protonated saccharide species and their transitions in time, and to calculate their finite-temperature anharmonic vibrational spectrum. Together, these enable the role of temperature on the conformer population and its dynamics as well as on the vibrational spectrum, to be taken into account.

All AIMD simulations were performed using the CP2K suite of programs. The electronic structure was calculated 'on-the-fly' using the Quickstep module ${ }^{53}$ that employs a hybrid Gaussian and plane wave approach to Kohn-Sham density functional theory for $a b$ initio Born-Oppenheimer molecular dynamics. $\underline{54}$ 5ㅡㄴ Though, until now, AIMD simulations of saccharide ions in the gas phase have mostly employed the BLYP-D functional $\stackrel{27,35-40}{ }$ this study opted for use of the B3LYP-D functional. B3LYP-D is generally believed to be more accurate than BLYP-D or similar functionals, in particular for $_{\text {ticle }}$ Online

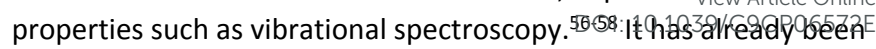
observed that IR spectra generated with the BLYP functional show a systematic underestimation of vibrational bands leading to redshifts of up to $100 \mathrm{~cm}^{-1}$ in some cases, ${ }^{59}$ (though many using BLYP out of computation time considerations have obtained good agreement with experiment $\underline{27}, \underline{34}, \underline{40}, \underline{59})$. Although computationally relatively costly, B3LYP is feasible for the systems studied here. B3LYP-D was selected as a reasonable compromise between accuracy and computational efficiency. The need for the latter renders higher level methods impracticable in the present state-ofthe-art due to the large computation time needed for AIMD calculations. A 6-31++G $(d, p)$ basis set was used with an auxiliary plane wave basis set defined by a 350 Ry electron density grid. Goedecker-Teter-Hutter atomic pseudopotentials optimized for the BLYP functional were used to represent the interaction between valence and core electrons. ${ }^{60}$ Matrix elements smaller than $10^{-12}$ Hartree/atom were neglected and the convergence criterion of the SCF wavefunction was set at $10^{-6} \mathrm{Hartree/atom}$. The default values for the Martyna-Tuckerman Poisson solver ${ }^{61}$ were used to establish non-periodic boundary conditions. The motion of the nuclei was evaluated classically using the velocity Verlet algorithm with a $0.5 \mathrm{fs}$ time step.

The simulations commenced with a 5 ps equilibration at $\sim 350 \mathrm{~K}$ in an NVT ensemble using the Nosé-Hoover Thermostat $\underline{62}, \underline{63}$ to maintain the system temperature. Conformers identified in the equilibration phase were optimized and subject to equilibration under the same conditions so as to form a group of conformers that persist at the experimental temperature of $\sim 350 \mathrm{~K}$. Following equilibration, 10 ps simulations were run for constant energy ensembles at an average temperature of $\sim 350 \mathrm{~K}$ for each of these conformers. Experience has shown that at high temperatures, $T>$ $300 \mathrm{~K}$, ten picoseconds is an adequate period for exploring the conformational space in system of this size. $\underline{27}, \underline{39}, \underline{40}$ The resolution of spectra was improved by extending the simulations to $20 \mathrm{ps}$, when deemed necessary. For better sampling of structures in time, ten trajectories were run for each conformer.

\subsubsection{Anharmonic Vibrational Finite Temperature Spectra from} AIMD Calculations: The use of AIMD calculations to compute vibrational spectra takes advantage of the thorough sampling of conformer space that occurs as the system traverses the potential energy surface during the simulation. In this way, static single conformer vibrational spectra at $0 \mathrm{~K}$ are replaced by calculated spectra that inherently consider multiple energetically relevant conformers and finite simulation temperature. Thus vibrational spectra calculated from AIMD simulations are well suited to systems where multiple conformers are expected to contribute significantly such as when modelling the bulk phase or high temperature systems. IR spectra are calculated from AIMD simulations by computing the autocorrelation function of the molecular dipole moment that is proportional to IR absorption coefficient. ${ }^{64}$ The Fourier transform of the autocorrelation function of the time derivative of the dipole moment permits the switch between the time and frequency domain where the lineshape of the IR spectrum is given as:

$A(\omega) \propto \int<\dot{\mu}(\tau) \dot{\mu}(t+\tau)>e^{-i \omega t} d t$ 
Where $A(\omega)$ is the infrared absorption coefficient and $<\dot{\mu}(\tau) \dot{\mu}$ $(t+\tau)>$ is the autocorrelation function of the time derivative of the dipole moment. This autocorrelation function is then obtained using the Wiener-Khintchine $\underline{65}, \underline{66}$ theorem as:

$$
<f(\tau) f(t+\tau)>=\left.\frac{1}{2 \pi}\left|\int\right| \int f(t) e^{-i \omega t} d t\right|^{2} e^{i \omega t} d \omega .
$$

Where the autocorrelation of a function $f(t)$ is equal to the application of the inverse Fourier transform on the square of the absolute value of the Fourier transform of $f(t)$. The fast Fourier transform algorithm is then used to improve the scaling from $n^{2}$ to $n \log (n) .67$

Calculation of IR spectra from AIMD simulations yields the anharmonic IR spectrum as the method does not require any assumption about the shape of the potential energy surface. However, other sources of anharmonicity that are purely quantum mechanical in origin such as the mode couplings which produce combination bands and Fermi resonances are not addressed by the method due to the reliance on classical dynamics. The use of classical dynamics to describe a molecular system is the source of the most serious drawback of the method. Problematically, quantum mechanical phenomena such zero point energies and interference effects are necessarily omitted in the classical model. However, when high temperature systems are considered the classical model is a more accurate description of the system and fewer discrepancies due to quantum effects are expected. In the present study, the anharmonic vibrational spectra at an average temperature of $\sim 350 \mathrm{~K}$ were calculated from the Fourier transform of the system dipole moment correlation function computed over the course of the simulations. System dipole moments were calculated at each time step using the Berry phase representation ${ }^{68}$ with calculation of the dipole moment of the box cell. The system dipole moments were calculated at each time step over a unit cell with a side dimension of $20 \AA$. The Fourier transform of the dipole moment time correlation function was averaged over ten trajectories to obtain the vibrational spectrum of each conformer. Individual spectral transitions were assigned by matching the Fourier transform of the time dependence of the bond length of the selected group of atoms participating in the transition to the transitions observed in the vibrational spectrum.

\subsubsection{Anharmonic Vibrational Spectra at $\mathbf{0} \mathrm{K}$ from VSCF}

Calculations: The VSCF method is also well suited to obtain anharmonic IR spectra. In this case, where the experimental spectra were recorded at high temperature, VSCF calculations complement the AIMD computed spectra. Here, quantum VSCF line spectra are used together with classical finite temperature AIMD spectra to help unravel the structure-spectra relations of the experimental species. VSCF treats anharmonic effects including those couplings between different normal modes using a fully quantum mechanical model. It is a mean field method where each vibrational mode moves in the mean field of all vibrational motions and is based upon the assumption of separability of the vibrational wavefunction, where the $\mathrm{N}$ mode wavefunction $\Psi$ is approximated as:

$\Psi\left(Q_{1}, \ldots, Q_{N}\right)=\prod_{i=1}^{N} \psi_{i}^{(n)}\left(Q_{i}\right)$.
Mass-weighted normal coordinates $Q_{1} Q_{2} \ldots$ for single-modetericle Online wavefunctions $\boldsymbol{\psi}_{i}^{(N)}$ are then used to formulate the. Shrole-pFode $572 \mathrm{E}$ vibrational Schrödinger equation: ${ }^{69-71}$

$\left[-\partial^{2} / 2 \partial Q_{i}^{2}+\bar{v}_{i}^{(n)}\left(Q_{i}\right)\right] \psi_{i}^{(n)}=\varepsilon_{i}^{(n)} \psi_{i}^{(n)}\left(Q_{i}\right)$

Here, the effective potential $\overline{\boldsymbol{v}}_{\boldsymbol{i}}^{(\boldsymbol{n})}\left(\boldsymbol{Q}_{\boldsymbol{i}}\right)$ for mode $\boldsymbol{Q}_{\boldsymbol{i}}$ is given by:

$\bar{v}_{i}^{(n)}\left(Q_{i}\right)=\left\langle\prod_{j \neq i}^{N} \psi_{j}^{(n)}\left(Q_{j}\right)\left|v\left(Q_{1}, \ldots, Q_{N}\right)\right| \prod_{j \neq i}^{N} \psi_{j}^{(n)}\left(Q_{j}\right)>\right.$

Equations (4) and (5) are solved self-consistently for the singlemode wavefunctions, energies and effective potentials of the individual modes to yield the anharmonic frequencies and intensities of single conformers at $0 \mathrm{~K}^{43}$ For efficiency ${ }^{72}$, the VSCF potential was approximated as a sum of terms which includes single-mode potentials and pair-wise interactions between normal modes:

$\boldsymbol{v}\left(Q_{1}, \ldots, Q_{N}\right)=\sum_{i=1}^{N} \boldsymbol{v}_{i}^{\text {diag }}\left(\boldsymbol{Q}_{i}\right)+\sum_{j} \sum_{i>j} \boldsymbol{W}_{i j}^{\text {coup }}\left(\boldsymbol{Q}_{i}, \boldsymbol{Q}_{j}\right)$.

Here, the single-mode diagonal terms $\boldsymbol{v}_{\boldsymbol{i}}^{\text {diag }}\left(\boldsymbol{Q}_{i}\right)$ are defined as:

$v_{i}^{\text {diag }}\left(Q_{i}\right)=\left(0, \ldots Q_{i}, \ldots 0\right)$,

And the pair-wise interactions as defined as follows:

$W_{i j}^{\text {coup }}\left(Q_{i}, Q_{j}\right)=v\left(0, \ldots Q_{i}, \ldots, Q_{j}, \ldots 0\right)-v_{i}^{\text {diag }}\left(Q_{i}\right)-v_{j}^{\text {diag }}\left(Q_{j}\right)$

Thus, the VSCF potential will account for anharmonicities that arise due to coupling between vibrational mode pairs. In this form, the potential is calculated by multidimensional grid point calculations. Both the anharmonic values of the $a b$ initio potential method and the dipole moment are calculated over a corresponding grid.

Besides having better scaling behaviour than representations based on quartic force-fields ${ }^{72}$, the pairwise interaction representation also allows one to consider which pairs of modes to include in the calculation, where only couplings between pairs of modes for hydrogenic vibrational transitions were calculated in this study so as to reduce the computation time. Although only the $\mathrm{OH}$ modes fell within the range of this experiment, both $\mathrm{OH}$ and $\mathrm{CH}$ modes were included in the calculation as the anharmonicity for hydrogenic transitions is relatively large. ${ }^{73,74}$ The VSCF-PT2 variant introduces a second-order perturbation correction to VSCF that has been demonstrated to improve accuracy. ${ }^{72,75}$ However, this approach is foregone in the present study in favour of VSCF due to the possible unsuitability of VSCF-PT2 in the case of degenerate vibrational states, something which cannot be discounted in the vibrational spectrum of a monosaccharide. It is important to understand that in cases where resonances are present, VSCF-PT2 may break down. Here however, good agreement between the method and a large number of experimental transitions indicates that VSCF contends well with any resonances present in this experimental system.

In realistic applications to all but the smallest systems, VSCF assumes a well-defined local minimum of the species, and treats the system in normal mode coordinates, but beyond the harmonic 
approximation. Thus, when the vibrations are not confined to the vicinity of a single minimum, VSCF may not be valid. On the other hand, the full quantum treatment gives it an advantage over AIMD. Accordingly, VSCF has shown great success in predicting the experimental spectra of polyatomic molecules under cold beam conditions, where the VSCF spectrum compares favourably to the well resolved experimental spectrum. ${ }^{49,}$ 50, 76

VSCF calculations of the anharmonic frequencies and intensities of the vibrational transitions of optimized conformer structures at 0 $\mathrm{K}$ were performed with the GAMESS program suite. ${ }^{77}$ Here again the B3LYP-D functional was employed along with the $6-31++G(d, p)$ basis set so as to provide a comparison between the calculated spectra from the AIMD simulation that included contributions from conformer dynamics at $\sim 350 \mathrm{~K}$ and line spectra derived from VSCF calculations of individual conformer structures at $0 \mathrm{~K}$. The VSCF derived spectra, while static and lacking finite-temperature fluctuations in conformer structure, serve to provide spectra from a fully quantum-mechanical model that does account for quantum effects such as zero point energy. These effects are absent in the AIMD method where classical dynamics is necessary. At the same time, AIMD simulation helps to evaluate the validity of VSCF for a given system by identifying if other nearby local minima are present, in which case a single-structure method such as VSCF would be inaccurate.

The interpretation of the IRMPD spectra was based on the vibrational calculations, where the calculated spectra of low energy conformers of protonated methyl galactopyranoside, galactosyl oxocarbenium ions and protonated anhydrogalactoses were compared with the experimental spectra. In this way, the presence of this group of low energy structures was evaluated under the conditions of the IRMPD experiment. The advantage gained with the use of both the VSCF and AIMD methods will become evident in the analysis of the experimental data.

\subsection{Experimental Methods}

Methyl- $\alpha$-D-galactopyranoside and methyl- $\beta$-D-galactopyranoside are available commercially (Sigma-Aldrich) and were used without further purification. The analytes were dissolved in $\mathrm{CH}_{3} \mathrm{OH}: \mathrm{H}_{2} \mathrm{O}=1: 1$ with $0.1 \%$ of acetic acid at a concentration of $5.10^{-5} \mathrm{~mol}^{-\mathrm{I}^{-1}}$ and were sprayed with conventional ESI conditions.

Infrared spectroscopy integrated to mass spectrometry was performed using a 7 Tesla Fourier transform ion cyclotron resonance (FT-ICR) tandem mass spectrometer (Bruker Apex Qe) coupled with IR lasers. ${ }^{78}$ The $\mathrm{m} / \mathrm{z} 163$ and 195 ions of interest were mass selected in the quadrupole and then accumulated in a hexapole ion trap. Ions were then pulse-driven into the ICR cell where they were subjected to IR irradiation.

Infrared spectra in the $900-1800 \mathrm{~cm}^{-1}$ and $3200-3700 \mathrm{~cm}^{-1}$ spectral ranges were recorded using the free electron laser (IR FEL) CLIO (Orsay, France) $)^{79}$ and an optical parametric oscillator/amplifier (OPO/OPA from LaserVision pumped by an Innolas Spitlight 600 running at $25 \mathrm{~Hz}$ ), respectively. Details about these experimental setups can be found elsewhere. ${ }^{78,80}$ The IR FEL delivers trains of $\sim 500$ picosecond pulses at $25 \mathrm{~Hz}$, and the laser mean power was roughly linearly decreasing from $1000 \mathrm{~mW}\left(900 \mathrm{~cm}^{-1}\right)$ to $450 \mathrm{~mW}$ $\left(2000 \mathrm{~cm}^{-1}\right)$. One or two attenuators $(-3 \mathrm{~dB}$ each) were necessary to avoid saturation when the IR FEL laser was tuned on ressonange wwith

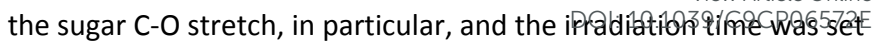
to $500 \mathrm{~ms}$. The typical output energy of the OPO/OPA is $\sim 12-13$ $\mathrm{mJ} /$ pulse at $3600 \mathrm{~cm}^{-1}$, but rapidly decreases at lower wavenumbers. A significant enhancement of the fragmentation signal could be observed using an auxiliary $\mathrm{CO}_{2}(10$ watt $\mathrm{CW}, \mathrm{BFi}$ OPTiLAS, France). As described previously, ${ }^{81}$ ions are irradiated with a $\mathrm{CO}_{2}$ pulse following each OPO/OPA pulse delivered at $25 \mathrm{~Hz}$, the delay being on the order of $\sim 1 \mu \mathrm{s}$. Experimental IR spectra correspond to the plot of the fragmentation yield, i.e. $-\ln [\mathrm{P} /(\mathrm{P}+\mathrm{F})]$ where $P$ and $F$ are the abundance of the parent and photofragments, as a function of the laser wavelength.

\section{Results and discussion}

\subsection{Spectroscopy and Dynamical Analysis of $m / z 195$ Species}

The protonated parent ion from methyl D-galactopyranoside has a mass of 195 amu and an $m / z 195$ peak was detected for both $\alpha$ - and $\beta$-anomers of methyl D-galactopyranoside, such that two IRMPD spectra for the $m / z 195$ species could be compared with each other as well as with the calculated spectrum. The experimental IRMPD spectra of the $m / z 195$ species from $\alpha$ - and $\beta$-anomers of methyl $D$ galactopyranoside along with the calculated spectrum of the lowest energy conformer of methyl $\beta$-D-galactopyranoside, appear in Fig. 2. The vibrational assignments for the calculated spectrum are also shown. As seen in the figure, the experimental spectra from the $\alpha$ and $\beta$-anomers of methyl $D$-galactopyranoside are nearly identical. This indicates that the anomeric identity of the protonated parent species is not resolvable in the portions of the mid- and near-IR spectrum obtained in the IRMPD experiment. This result may be due to the inherent difficulty in resolving the anomeric configuration of the protonated glycoside at the thermal conditions of the IRMPD experiment. ${ }^{82}$ However, an alternative explanation involving interconversion of $\alpha$ - and $\beta$-anomers during ESI which was previously demonstrated for protonated galactosamine ${ }^{83}$, is also a possibility.

The simulations were initiated at the equilibrium geometry of the lowest energy conformer of protonated methyl $\beta$-Dgalactopyranoside. A complete discussion of higher energy conformer spectra will be treated in a forthcoming study. The closely similar profiles of the calculated and experimental spectra encourage its assignment to the $m / z 195$ species isolated from methyl $\alpha$ - and $\beta$-D-galactopyranoside in the IRMPD experiment. The large blue-shift, $\sim 200 \mathrm{~cm}^{-1}$ of the calculated vibrational bands in the near-IR region relative to the intensity maxima of the experimental spectra may be associated with the B3LYP-D functional itself or its combination with the modest sized $6-31++G(d, p)$ basis set. The neglect of zero point energy contributions in the classical calculations may also be a source of error, particularly for the $\mathrm{OH}$ stretches for which the zero point energy contribution is substantial. In either case a very floppy system of this size is a challenge for AIMD spectra calculation. Although the use of B3LYP does give accurate vibrational band frequencies when spectra are computed with the VSCF method, $, 99,56,58$ see Fig. 7 , the situation may be different in the context of MD simulation since trajectories may involve large excursions away from equilibrium where the B3LYP potentials may be less accurate. 
Further support for this assignment is based upon the most notable feature of the lowest energy conformer of $\mathrm{BMeGal}^{\mathrm{H}} \mathrm{H}^{+}$ protonation at the hydroxymethyl oxygen, where a proton is shared between this oxygen and 04 , to form a second six-membered ring that is out-of-plane to the pyranose ring. ${ }^{27}$ (The same feature appears in the lowest energy $\alpha \mathrm{MeGal}-\mathrm{H}^{+}$conformer (which was only $0.008 \mathrm{eV}$ higher in energy than the lowest energy $\beta$-anomersee Fig. 1). The shared proton leads to a red-shift of $\sim 50-120 \mathrm{~cm}^{-1}$ of the intensity maxima in the near-IR OH stretching region away from the principle intensity maximum at $\sim 3656 \mathrm{~cm}^{-1}$. The protonated sugar presents the structure of an intramolecular proton-bound dimer. Previous studies of gas phase vibrational spectra for intermolecular proton-bound $\mathrm{O} . . . \mathrm{H}^{+} \ldots \mathrm{O}$ dimers, ${ }^{27,84,85}$ have identified a significant red-shift of the stretching transitions of the oxygens involved. The vibrational mode assignments of the calculated spectrum of $\beta \mathrm{MeGal}-\mathrm{H}^{+}$show similar behaviour: the $\mathrm{OH}$ stretches of $\mathrm{O} 4$ and $\mathrm{O} 6$ that do not involve the proton shared between them are red-shifted by $\sim 90-130 \mathrm{~cm}^{-1}$ from the $\mathrm{OH}$ stretches of $\mathrm{O} 2$ and $\mathrm{O} 3$ (see Fig. 2, bottom panel). This confirms that the oxygens flanking the shared proton are responsible for the shift of $\mathrm{OH}$ stretching transitions to lower wavenumber observed in the calculated spectrum and that a shared proton between $\mathrm{O} 4$ and O6 is responsible for the shift of intensity maxima to lower wavenumber in the near-IR region of the experimental spectra.

Evidence of shared proton binding is also present in the mid-IR region. The IRMPD spectra of methyl $\alpha$ - and $\beta$-D-galactopyranoside and the calculated spectrum of $\beta \mathrm{MeGal}-\mathrm{H}^{+}$all show a vibrational transition at $\sim 1600 \mathrm{~cm}^{-1}$. The vibrational mode assignments of the calculated spectrum of methyl $\beta$-D-galactopyranoside show that this transition includes a significant contribution from motions of the shared proton (see Fig. 2, bottom panel). This vibrational transition at $\sim 1600 \mathrm{~cm}^{-1}$ has been identified in the spectra of cold proton bound alcohol dimers where an asymmetric stretching band of the shared proton was observed at $1595 \mathrm{~cm}^{-1}$ for a $\mathrm{Me}_{2} \mathrm{O} \cdot \mathrm{H}^{+} \cdot \mathrm{HOMe}$ dimer and at $1638 \mathrm{~cm}^{-1}$ for its proton bound ethanol homolog. ${ }^{84}$ While the shared proton in the lowest energy conformer of $\mathrm{BMeGal}^{+} \mathrm{H}^{+}$is intramolecular dimer of the $\mathrm{RCHO} \ldots \mathrm{H}^{+} \ldots \mathrm{HOCH}_{2} \mathrm{R}^{\prime}$ type and the IRMPD spectra were obtained at high temperatures, the presence of the intensity maximum at $\sim 1600 \mathrm{~cm}^{-1}$ provides a third diagnostic indicating a shared proton motif in the experimentally isolated species.

The position of the shared proton between the hydroxymethyl oxygen and $\mathrm{O} 4$ is strongly supported by the dynamics simulation. During the $10 \mathrm{ps}$ simulation initiated from the lowest energy conformer of $\beta \mathrm{MeGal}-\mathrm{H}^{+}$, few significant structural changes occur such that only one stable conformer of protonated $\beta$ MeGal- $\mathrm{H}^{+}$ endures at $\sim 350 \mathrm{~K}$. This is the minimum energy structure is shown in Fig. 1a. A stable yet higher energy ( $\sim 0.5 \mathrm{eV})$ conformer also with a second six-membered ring between the hydroxymethyl oxygen and $\mathrm{O} 4$ but with clockwise hydroxyl groups at $\mathrm{C} 2$ and $\mathrm{C} 3$ was identified as well $\left(\mathrm{S}^{\dagger}+\right)$. However, simulations initiated from this higher energy structure yielded the lowest energy reversed conformer after only a few hundreds of femtoseconds. The stability of the reversed double six-membered ring conformer and the preferential protonation at the hydroxymethyl position of singly protonated methyl $\beta-D-$ galactopyranoside are both closely interlinked with the structure of the sugar. The axial hydroxyl group at $\mathrm{C} 4$ in galactose uniquely permits the formation of a six-membered ring which does pot significantly strain the pyranoside.

DOI: 10.1039/C9CP06572E

In conclusion, the strong similarity between the experimental spectra and the averaged calculated spectrum from the AIMD simulations indicate assignment of the $m / z 195$ species to the lowest energy conformer of $\beta \mathrm{MeGal}-\mathrm{H}^{+}$. Energetic considerations as well as agreement between the experimental and calculated vibrational transitions suggest that protonation of the glycopyranoside between the hydroxymethyl group and axial hydroxyl group creates an intramolecular proton shared dimer. The proton-bound dimer was an enduring feature through the AIMD simulation, and its presence can be expected to have implications regarding proton transport within the sugar which are relevant to the microscopic mechanisms of acid catalysed reactions of glycopyranosides.

\subsection{The $m / z 163$ Species: Conformational Transitions, Vibrational Spectroscopy and Dynamical Analysis}

3.2.1 Conformational Transitions of $m / z 163$ species: In contrast to simulations of MeGal- $\mathrm{H}^{+}$which demonstrated the stability of the protonated sugar at the experimental temperature, cations investigated as possible structures for the $m / z 163$ species were found to transition between multiple comformers with short lifetimes. In order to begin the computational search for a $m / z 163$ species derived from methyl $\alpha$ - and $\beta$-D-galactopyranoside, two archetypal protonated species possessing this mass-to-charge ratio were considered: a galactosyl oxocarbenium ion and a protonated anhydrogalactose. Fig. 3 presents the structures and relative energies of the low energy conformers of these $m / z 163$ species, as well as the transitions between the structures observed during AIMD simulation. Ring puckering of the pyranosides is described using the Cremer-Pople puckering coordinates ${ }^{86}$ and the orientations about the $\mathrm{C} 4 \mathrm{O} 4, \mathrm{C} 5 \mathrm{C} 6$ and $\mathrm{C} 6 \mathrm{O} 6$ bonds are also noted in the figure. The low energy conformer search for the galactosyl

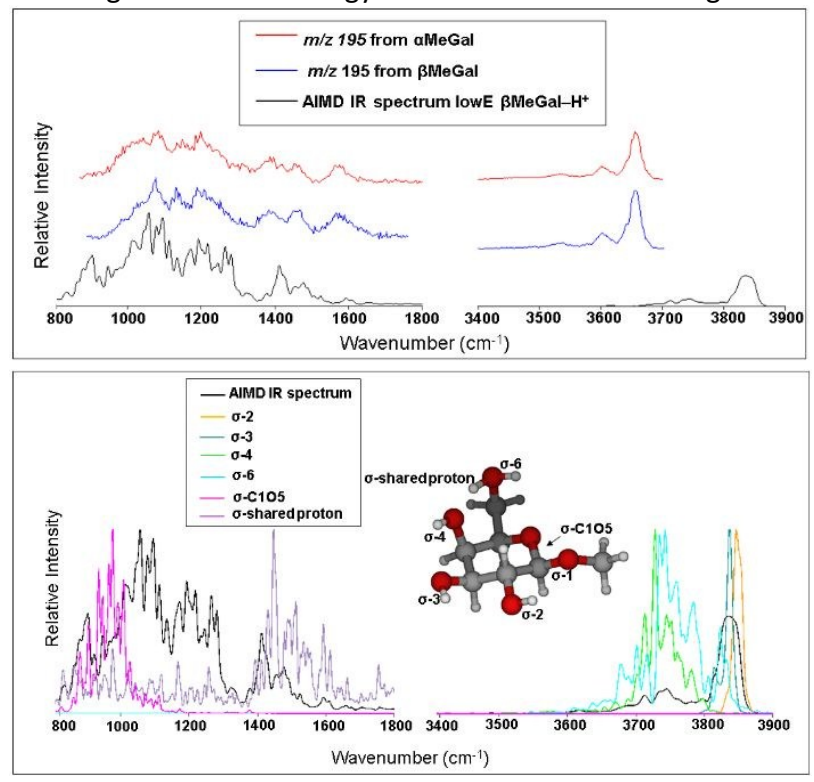

Fig. 2 (top panel) Comparison of the IRMPD spectra of the $m / z 195$ species with the averaged spectrum calculated from AIMD simulations of the lowest energy conformer of $\beta \mathrm{MeGal}-\mathrm{H}^{+}$. (bottom panel) Assignment of individual vibrational modes against the backdrop of the averaged calculated spectrum. The modes are distinguished by colour. 
oxocarbenium ion (I) was initiated from the minimized structures of protonated methyl D-galactopyranoside from which the methanol moiety had been cleaved. Optimization of these structures at the B3LYP-D/6-311++G $(d, p)$ level yielded two distinct conformers with a relative energy difference between them of $0.470 \mathrm{eV}(\mathrm{S} 2+)$. The search for low energy conformers of protonated 1,6-anhydro- $\alpha$-Dgalactofuranose (II) and levogalactosan (III) began from the neutral optimized structures of II and III, respectively, with a proton placed 1.9 Å from oxygens O2-O6 (identified in Fig.1). Optimization was carried out at the B3LYP-D/6-31++G (d,p) level for each oxygen position to identify the energetically preferred protonation sites of the anhydrogalactoses. It was found that protonation of $\mathrm{O} 6$ in both II and III yielded the lowest energy conformers. Strikingly, this protonated galactosan was the lowest energy conformer in the system and was $0.237 \mathrm{eV}$ below the lowest energy conformer of I and fortuitously, the lowest energy conformer of II (see Fig. 3, top panel).

The search for low energy conformers of galactosyl oxocarbenium ion and protonated anhydrogalactoses was continued with AIMD simulation. NVT ensemble simulations were used to equilibrate the system at $350 \mathrm{~K}$ with trajectories of $5 \mathrm{ps}$ duration. These trajectories were initiated from the two minimum energy structures of $\mathrm{I}$, the minimum energy structure of II and a high energy optimized structure of III. Additional conformers identified during the equilibration phase were in turn optimized and used to initiate NVT simulations. Trajectories at $\sim 350 \mathrm{~K}$ were taken from the equilibration phase to initiate simulations of NVE ensembles from which conformer lifetimes, relative populations, transitions and IR spectra were recorded. In this way it was found that while the lower energy conformer of I remained unchanged during the simulations, the higher energy conformer $(+0.470 \mathrm{eV})$ decayed within a few 100 femtoseconds to lower energy conformers $\left(\mathrm{S}^{+}\right)$. These six lower energy conformers included the lower energy galactosyl oxocarbenium ion previously identified by optimization (0.258 eV) and a non-oxocarbenium conformer (IV) with a protonated 1,4-Anhydro- $\beta$-D-galactopyranose structure $(0.319 \mathrm{eV})$. The five low energy conformers of I and single conformer of IV exhibited transitions between one another. Though some transitions occurred at relatively high frequency ( $\geq 25$ transitions within a $400 \mathrm{ps}$ simulation time considering all trajectories), other transitions occurred as rare events that were observed only once throughout the simulations (see Fig. 3, top and middle panels). Often, each species would only live a few thousand fs before transitioning as shown in the representative snapshots from an NVE simulation trajectory seen in the bottom panel of Fig. 3. As the energetic proximity of the conformers was less than 0.1 $\mathrm{eV}$, the occurrence of the transitions during high temperature simulations was not surprising. However, as Fig. 3 shows, the most enduring conformers were not necessarily of the lowest energy indicating the existence of significant energy barriers. Accordingly, the middle panel of Fig. 3 identifies the predominant conformers as those of the lowest energy $(0.237$ and $0.258 \mathrm{eV})$ but the third most prominent was at the highest energy $(0.340 \mathrm{eV})$. (The conformer with a relative energy of $0.276 \mathrm{eV}$ was not used to initiate NVE simulations from which vibrational spectra could be calculated as its short lifetime indicated that it was not a large contributor to the spectrum).
The NVT ensemble simulation initiated from the minimumaenergy line

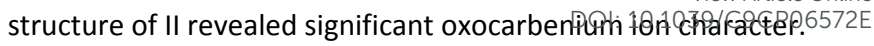
During the course of the simulation the bond between $\mathrm{C} 1$ and $\mathrm{O} 6$ was lengthened to be between 2.00 and $2.74 \AA$ for prolonged (up to $0.9 \mathrm{ps)}$ and repeated periods. No additional stable conformers of II were found. In contrast, the high energy conformer of III quickly ( 1 ps) transitioned to its lowest energy conformer (S4+). Once formed, no further transitions were observed for this low energy structure. The energetic difference between this conformer and higher energy conformer of III was at least $0.366 \mathrm{eV}$, such that transitions to the starting structure would be energetically prohibitive as would be transitions to the galactosyl oxocarbenium ion conformers which are at least $0.237 \mathrm{eV}$ higher in energy. NVE ensemble simulation of the lowest energy conformer of I and the lowest energy conformers of II and III were extended to 20 ps to improve the resolution of calculated spectra for these lowest energy species.
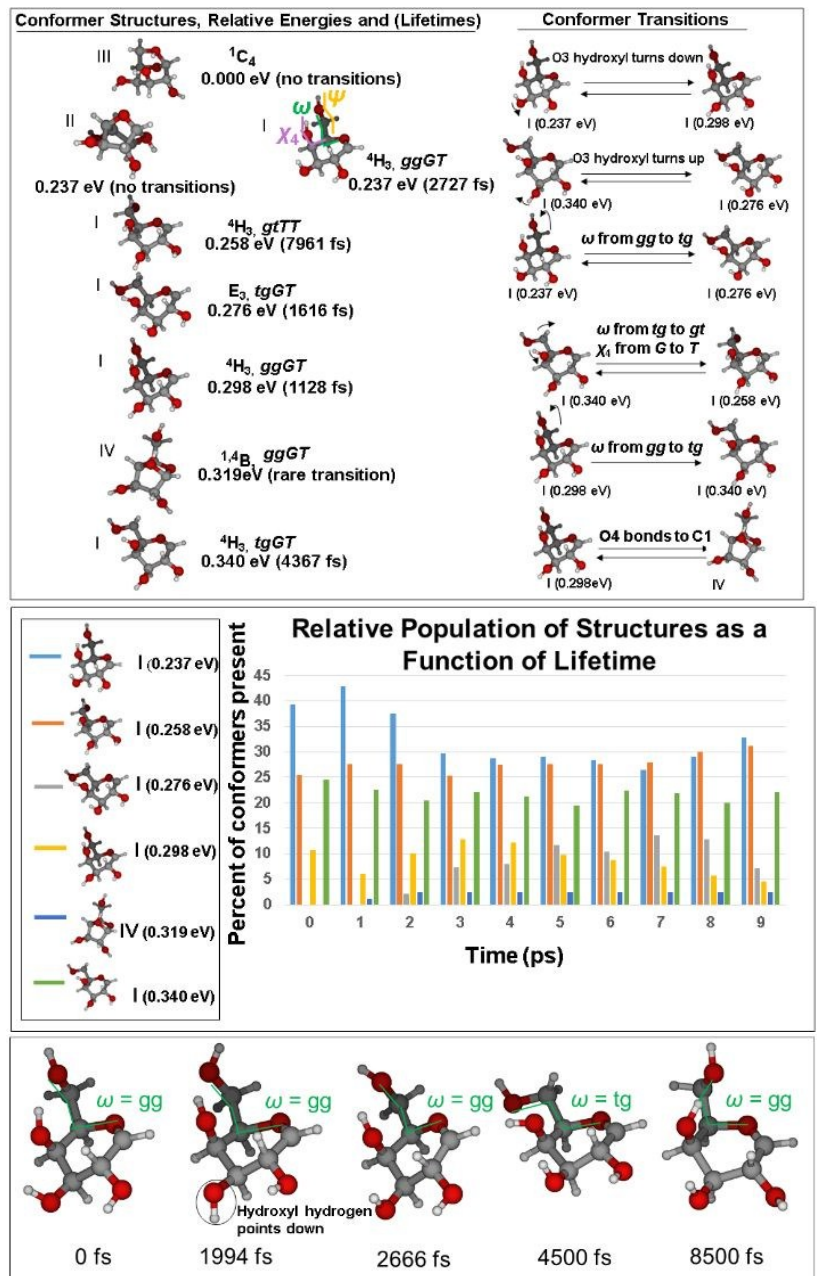

Fig. 3 (top panel) Optimized structures of I, II, III and IV observed in AIMD simulations. The relative energies and lifetimes of the conformers are noted. Transitions between conformers are described. (middle panel) The relative presence of conformers of $I$ and IV over time during AIMD simulations. (bottom panel) Snapshots of conformers over a single NVE trajectory. Changes in the angle $\omega$ and the position of hydrogen in the 03 hydroxyl group are noted. The angles $\omega, \psi$ and $\chi_{4}$ are defined in the supplementary information according to the usage in the literature ${ }^{87}$. 
The main finding from dynamics simulations of the galactosyl oxocarbenium ion and protonated anhydrogalactose is that there are conformers of very different lifetimes. While some conformers seem to be quite stable due to appreciable barriers against transition, most conformers were shown to have exceedingly short lifetimes. When frequent conformer transitions proved to be the rule, single conformer VSCF calculations cannot accurately predict experimental spectra. This finding affected vibrational spectroscopy calculations as will be seen in the next section.

3.2.2 Vibrational Spectroscopy of $m / z 163$ species: Calculated vibrational spectra of the galactosyl oxocarbenium ion conformers and protonated anhydrogalactoses were computed from AIMD and VSCF calculations. The vibrational spectra calculated from AIMD simulations are compared with the IRMPD spectra of the $m / z 163$ species obtained from $\alpha$ - and $\beta$-anomers of methyl $D$ -

galactopyranoside in Fig. 4. In this way, the IRMPD spectra were compared with both higher resolution spectra calculated from 20 ps simulations of the lowest energy conformer of I, II and III and lower resolution spectra calculated from 10 ps simulations of three other low energy conformers of I and a single conformer of IV.

As in the case of the IRMPD spectra of the $m / z 195$ species (see section 3.1), the experimental spectra of the $m / z 163$ species are identical. Thus, any possible differences between the $\mathrm{m} / \mathrm{z} 163$ species that originate in the anomericity of the parent species, protonated methyl D-galactopyranoside, are not resolved under the conditions of the experiment. Therefore this study is unable to determine the influence anomericity plays in the formation of the $\mathrm{m} / z 163$ species in the gas phase. The spectra in this work do, however, address the influence of intramolecular hydrogen bonding on the gas phase vibrational spectra of glycosyl oxocarbenium ions. Previous studies of gas phase glycosyl oxocarbenium ion spectra have been limited to ions with protecting groups. $\underline{26}, \underline{31}$ Studies of protected oxocarbenium ions advance the understanding of oxocarbenium ion reactivity most relevant to contemporary synthesis strategies ${ }^{35}$, yet do not address intramolecular hydrogen bonding- the dominant factor determining the conformational stability of isolated monosaccharides. ${ }^{88-90}$ Accordingly, Fig. 4 exhibits the unique calculated spectra of four conformers of galactosyl oxocarbenium ion all of which share a single puckering coordinate $\left({ }^{4} \mathrm{H}_{3}\right.$, see Fig. 3 top panel). This suggests that when intramolecular hydrogen bonding is present, the puckering coordinate is insufficient to describe the spectra, as was previously shown for gas phase rotational spectra of neutral sugars. ${ }^{91}$

A comparison between the experimental and calculated spectra reveals that two vibrational transitions occur in the near-IR region of the IRMPD spectra. However, only the calculated spectra of the protonated levogalactosan and the lowest energy conformer of I ( $0.237 \mathrm{eV})$ and its $\mathrm{C} 3$ rotamer $(0.298 \mathrm{eV})$ displayed two vibrational transitions in this high wavenumber transition region. The origin of this moderate intensity vibrational transition at $\sim 3750 \mathrm{~cm}^{-1}$ appears to be the presence of a moderate strength hydrogen bond in the cation. Fig. 5 groups the low energy conformers of I and the lowest energy conformers of II, III and IV according to the number of nearIR transitions in the calculated spectra and notes the length of hydrogen bonds in each species. With the exception of the strong hydrogen bond in IV, it can be seen that the only species with two

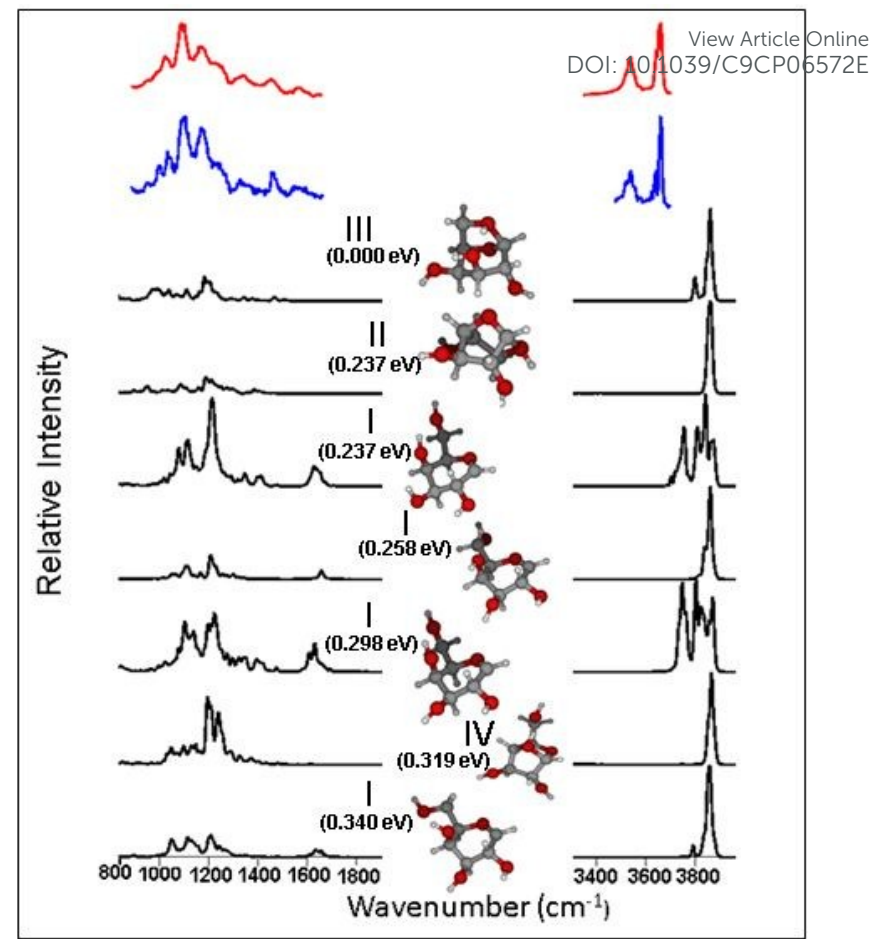

Fig. 4 IRMPD spectra of $m / z 163$ species from methyl $\alpha$-(in red) and $\beta$ - (in blue) Dgalactopyranoside compared with the calculated AIMD spectra of protonated

levogalactosan, protonated 1,6-Anhydro- $\alpha$-D-galactofuranose, low energy conformers of galactosyl oxocarbenium ion and protonated 1,4-Anhydro- $\beta$-D-galactopyranose (in the order of their relative energies). The AIMD calculated spectra all exhibit large blueshifts of $\sim 200 \mathrm{~cm}^{-1}$ in the near-IR region as noted in section 3.1 .

near-IR vibrational transitions have significant hydrogen bonding. The strong hydrogen bond in IV is seen however to give rise to an even more extensive red-shift. Consideration of the vibrational mode assignment of the species given in Fig. 6 shows that the very strong hydrogen bonds in the lowest energy conformers of III and IV are red-shifted into the $\mathrm{CH}$ stretch region of the spectrum. Thus, only the red-shifted $\sigma-3$ stretch of the lowest energy conformer of III and the moderately strong hydrogen bonding interactions in the lowest energy conformer of I and its C3 rotamer could be responsible for the observed transition at $\sim 3750 \mathrm{~cm}^{-1}$ in the IRMPD spectra.

The vibrational transitions in the mid-IR region also support the likeness between the calculated spectra of the lowest energy conformer of I and its C3 rotamer to the IRMPD spectra. The mid-IR region of the IRMPD spectra is characterized by high intensity vibrational bands between $1080-1240 \mathrm{~cm}^{-1}$. These transitions are featured only in the spectra of the lowest energy conformer of I and its $\mathrm{C} 3$ rotamer (see Fig. 4). As these high intensity transitions as well as the $3750 \mathrm{~cm}^{-1}$ vibrational band are unique to the calculated spectra of the lowest energy conformer of I and its C3 rotamer, it is likely that the moderately strong hydrogen bonding interactions between $\mathrm{O} 4$ and 06 are responsible for both spectral features. Further characterization of the experimental species is made possible by noting that the spectrum of the $\mathrm{C} 3$ rotamer exhibits transitions of nearly equal intensity at $\sim 3750$ and $\sim 3860 \mathrm{~cm}$ 1.However, the spectrum of the lowest energy conformer of I shows an intensity ratio of 1:1.5 that is much closer to that of the IRMPD 


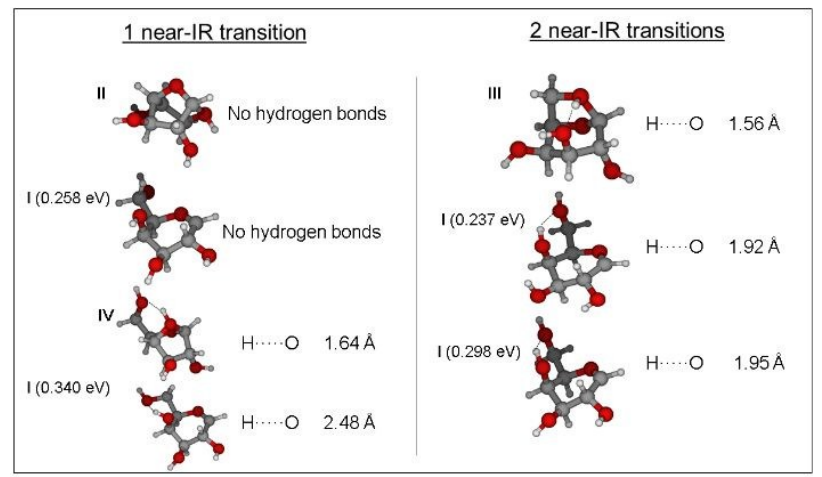

Fig. 5. Length of hydrogen bonding interactions in the low energy conformers of $\mathrm{I}$, and the lowest energy conformers of II, III and IV).

spectra (1:2 and 1:2.5 in the spectra from methyl $\alpha$ - and $\beta-D-$ galactopyranoside, respectively). This discrepancy along with the lower and decreasing relative population of the C3 rotamer (see Fig. 3) suggest that the lowest energy conformer of galactosyl oxocarbenium ion provides the best possible assignment of the experimental $m / z 163$ species among all the structures considered here based on the AIMD calculated spectra. However, multiple species may in fact be present under the experimental conditions. In this case, the presence of species such as I $(0.258 \mathrm{eV})$ and I ( 0.340 $\mathrm{eV}$ ) as well as the lowest energy conformers of II and III which lack intense vibrational transitions in the mid-IR region may be masked if their AIMD spectra are considered alone.

VSCF calculated anharmonic vibrational spectra of the equilibrium structures of the individual $m / z 163$ species at $0 \mathrm{~K}$ are presented in Fig. 7 (Table S1+) against the backdrop of the IRMPD spectrum of the $m / z 163$ species derived from $B$ methyl $D$ galactopyranoside. The transitions between conformers of I occurred frequently enough such that the longest conformer lifetime observed did not exceed 8 ps (see Fig. 3, top panel). Under these conditions a quasi-stationary system did not exist for the low energy conformers of I. In this case, VSCF calculations which yield the vibrational spectra of equilibrium structures would be expected to offer a less accurate depiction of the vibrational transitions of these short-lived conformers than AIMD derived spectra. In previous studies, ${ }^{92-95}$ the accuracy of VSCF has been shown to be

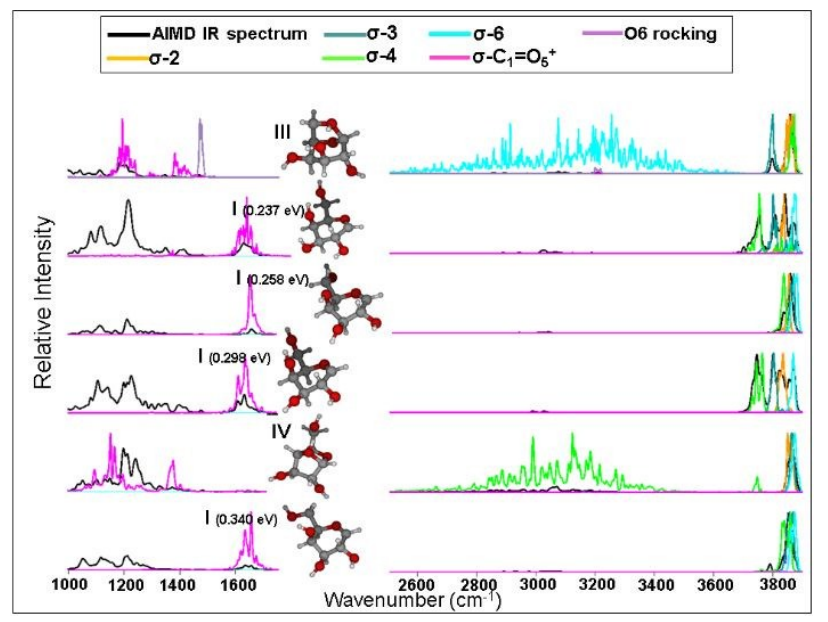

Fig. 6 The individual vibrational mode assignments of the calculated vibrational spectra. The vibrational modes are distinguished by colour. Note that the vibrational mode assignment of II has been omitted due to different connectivity.

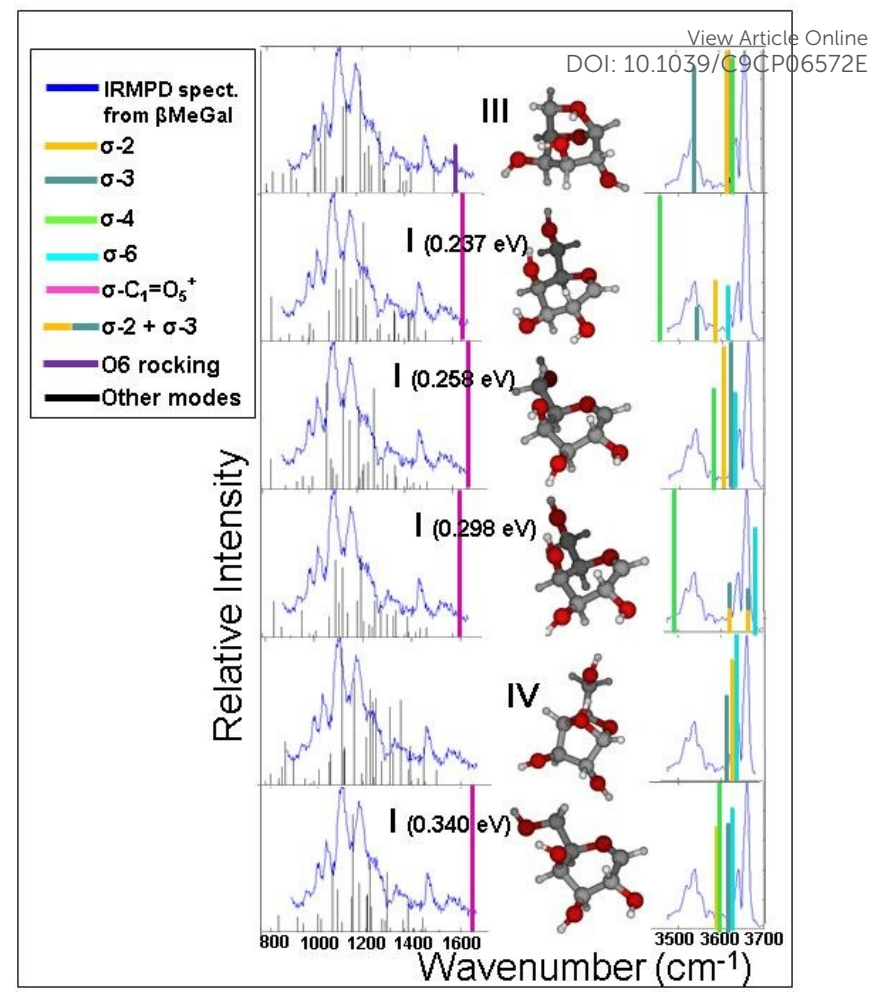

Fig.7 Comparison of the IRMPD spectrum of the $m / z 163$ species from methyl $\beta$-Dgalactopyranoside (in blue) with the calculated VSCF spectra protonated levogalactosan, low energy conformers of galactosyl oxocarbenium ion and protonated1,4-Anhydro-6-D-galactopyranose (in the order of their relative energies). Vibrational transitions are colour-coded.

within $40 \mathrm{~cm}^{-1}$ or less of the experimental vibrational transition. Lower accuracy than this is indicative of a system that does not meet the method's basic assumptions such as the presence of conformer transitions on the picosecond timescale. Indeed, Fig. 7 shows relatively poor agreement between the VSCF spectra of the galactosyl oxocarbenium ion conformers and the experimental spectrum but this caveat does not apply to the lowest energy conformer of III which did not transition to any other stable structure over the course of a 20 ps AIMD simulation, nor to I $(0.258 \mathrm{eV})$ or IV which do not readily transition to other $\mathrm{m} / \mathrm{z} 163$ species (see Fig. 3, middle panel). The VSCF spectrum of the lowest energy conformer of III is very similar to the experimental spectrum. Thus while the VSCF spectra cannot predict the experimental spectrum of the galactosyl oxocarbenium ion conformers and does not show significant agreement with the IRMPD spectra in the case of the protonated 1,4-Anhydro- $\beta$-Dgalactopyranose, the VSCF spectrum of protonated levogalactosan is in good agreement with the IRMPD spectrum. Additionally, there is reasonably good agreement between the VSCF spectrum of I $(0.258 \mathrm{eV})$ and the IRMPD spectra (see Table S1 ${ }^{\dagger}$ ) so as to suggest that this relatively stable conformer may also be among the $\mathrm{m} / \mathrm{z}$ 163 species. The lowest energy conformer of I transitions to the longest-lived galactosyl oxocarbenium ion conformer (see Fig. 3, top panel) so that is conformer is a significant contributor to the AIMD spectrum of I $(0.237 \mathrm{eV})$.In contrast, the VSCF spectrum of the lowest energy conformer of II is in poor agreement with the experimental spectrum $\left(\mathrm{S}^{+}\right)$, such that neither AIMD nor VSCF 
calculations suggest that it is significantly present among the experimental species.

Inspection of the VSCF spectrum of the lowest energy conformer of III shows that the $\mathrm{O} 6$ rocking mode transition occurs at $1588 \mathrm{~cm}^{-1}$. The proximity of the 06 rocking mode to the experimental vibrational transition at $\sim 1570 \mathrm{~cm}^{-1}$ suggests that an 06 rocking mode may be responsible for the highest wavenumber vibrational transition in the mid-IR region of the experimental spectrum. In this case, it is unclear if a characteristic $\sigma-\mathrm{C}_{1}=\mathrm{O}_{5}{ }^{+}$ stretch of the oxocarbenium ion previously reported for glycosyl cations ${ }^{26}$ at $1585 \mathrm{~cm}^{-1}$ is present in the IRMPD spectra of the $\mathrm{m} / \mathrm{z}$ 163 species. While the observed transition at $\sim 1570 \mathrm{~cm}^{-1}$ is reasonably close to the $\mathrm{O} 6$ rocking mode in the VSCF spectrum of the lowest energy conformer of III, the $\sigma-\mathrm{C}_{1}=\mathrm{O}_{5}{ }^{+}$transition of the lowest energy conformer of I occurs at $1630 \mathrm{~cm}^{-1}$ in both the AIMD and VSCF calculations (see Figs. 6 and 7, respectively). In the mid-IR region the VSCF spectrum, shown in Fig. 7, displays the harmonic transitions, as the anharmonicity is small in this region. Here, the calculated $\sim 1630 \mathrm{~cm}^{-1} \sigma-\mathrm{C}_{1}=\mathrm{O}_{5}{ }^{+}$stretch of the free galactosyl oxocarbenium ion is very close to the calculated $\sigma-\mathrm{C}_{1}=\mathrm{O}_{5}{ }^{+}$stretch for permethylated mannosyl oxocarbenium ion ${ }^{26}$ when the empirical scaling factor of the harmonic transition is neglected. While the unprotected and permethylated systems are not directly comparable, it seems that only the use of an empirical scaling factor could bring the calculated frequencies of the free galactosyl oxocarbenium ion in line with the experimentally observed transitions.

No such ambiguity exists for the VSCF spectrum of the lowest energy conformer of III, where the unscaled calculated 06 rocking mode is within $20 \mathrm{~cm}^{-1}$ of the experimental vibrational transition. Additionally, the lowest energy conformer of III is also energetically favoured as this species was at least $0.237 \mathrm{eV}$ lower in energy than any other $m / z 163$ species considered by this study. Further explanation is however necessary to address the significant differences between the AIMD and VSCF spectra of the lowest energy conformer of III. As the lowest energy conformer of III is stable during dynamics simulation (see the following section), differences between the spectra calculated by the two methods appear incongruous. If a single conformer is present during the simulation, it would be expected that its AIMD derived spectrum would closely resemble the VSCF single structure spectrum. However, while the VSCF spectrum depicts the vibrational transitions of the conformer's equilibrium structure at $0 \mathrm{~K}$, the high temperature of the simulation permitted large fluctuations away from the equilibrium structure (see Fig. 9). While these femtosecond fluctuations did not result in conformer transitions, it is likely that the relatively short duration of the trajectories caused them to contribute significantly to the AIMD spectrum due to insufficient statistics. In this case the VSCF spectrum of the lowest energy conformer of III may be closer to the observed vibrational transitions of the $m / z 163$ in the IRMPD spectra than its AIMD spectrum.

Therefore, the VSCF spectrum suggests that protonated levogalactosan may be present among the $m / z 163$ experimental species. This finding taken together with the good agreement between the IRMPD spectra and the AIMD spectrum of the lowest energy conformer of the galactosyl oxocarbenium ion indicates that both species are probably present under the conditions of $f_{A}$ the $e_{\text {Online }}$

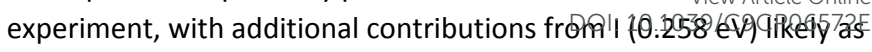
well. Indeed, assignments to 'static' structures may be misleading since molecular dynamics simulation reveals that the protonated levogalactosan also possesses oxocarbenium ion character (see the following section).

3.2.3 Molecular Dynamics of $m / z 163$ species: The dynamical analysis of galactosyl oxocarbenium ion structures treated in this study is dominated by the transitions between conformers as described in Fig. 3. While the $0.237,0.258$ and $0.340 \mathrm{eV}$ conformers were significantly longer lived than the 0.276 and $0.298 \mathrm{eV}$ conformers, all conformers exhibited at least one transition during the simulation. Fig. 8, shows the evolution of the dihedral angle $\omega$ (as defined in section 3.2.1) which depicts the conformational changes in the hydroxymethyl group as well as the $\mathrm{C} 105$ bond length in a representative trajectory of the lowest energy $(0.237 \mathrm{eV})$ conformer of $\mathrm{I}$. While transitions between the conformers of $\mathrm{I}$ occurred every few picoseconds as can be seen from the frequent changes in the angle $\omega$, the $\mathrm{C} 1 \mathrm{O} 5$ bond distance did not change significantly. Throughout the 20 ps simulation, a double bond is maintained at $\mathrm{C} 105$ such that the localization of the oxocarbenium ion's positive charge is not appreciably altered.

In contrast to the rapid transitions of the galactosyl oxocarbenium ion conformers, the protonated anhydrogalactoses exhibit a single stable conformation over 20 ps at $350 \mathrm{~K}$. At the same time, simulations of the lowest energy conformer of II and III show that these species possesses oxocarbenium ion character. Fluctuations in the length of the $\mathrm{C} 106$ bond in the lowest energy conformers of II and III are shown in Fig. 9, along with the changes

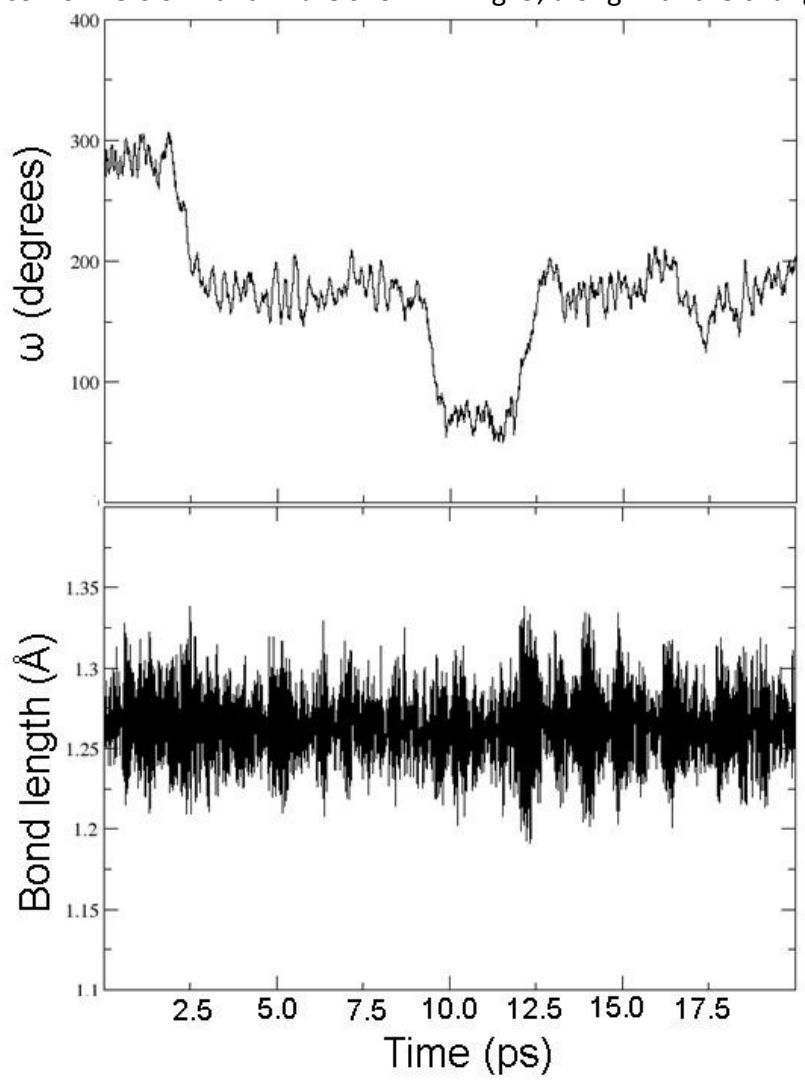

Fig. 8 Evolution of the bond distance at $\mathrm{C} 105$ and the dihedral angle $\omega$ of the $0.237 \mathrm{eV}$ conformer of $\mathrm{I}$. 
in the length of their respective $\mathrm{C} 1 \mathrm{O} 5$ bonds. The periodic shifts to non-bonding interactions between $\mathrm{C} 1$ and $\mathrm{O} 6$ that are accompanied by double bonding of the $\mathrm{C}=\mathrm{O}^{+}$type demonstrate significant oxocarbenium ion character in these species. This oxocarbenium ion character was especially pronounced in the lowest energy conformer of II where the oxocarbenium carbocation endured for $1.28 \mathrm{ps}$. The oxocarbenium ion character of the lowest energy conformers of II and III is thus demonstrated by dynamics and not by the presence of a spectral oxocarbenium ion signature.

Therefore, the protonated levogalactosan conformer that is likely present among the $m / z 163$ experimental species based on the good agreement of its VSCF spectrum with the IRMPD spectra is revealed to be in part an oxocarbenium ion species in the simulation. Furthermore, the oxocarbenium ion character of the anhydrogalactose cations demonstrates a stabilization mechanism for oxocarbenium ions of saccharides involving anhydro forms of the sugar cation under pyrolytic conditions. Previously, pyrolytic reactions of saccharides were thought to proceed through concerted displacement, as a mechanism for oxocarbenium ion stabilization that did not invoke solvent interactions was hitherto unknown. ${ }^{45}$ This study has shown the dual galactosan-

oxocarbenium ion nature of the levogalactosan cation, where the stable ion exhibits femtosecond periods of increased reactivity at the anomeric carbon.

Although, both II and III were formed in comparable yields under the conditions of acid-reversion and pyrolysis in vacuum, respectively, $\underline{44}, \underline{45}$ the gas phase cations of these anhydrogalactoses in the present study seem to favour protonated levogalactosan, which was $0.237 \mathrm{eV}$ more stable than the furanose. Interestingly, the hydrogen bond between $\mathrm{O} 6$ and $\mathrm{O} 3$ which is the source of protonated levogalactosan's remarkable stability (see Fig. 3) strongly evokes the 3,6 -Anhydro-D-hexoses proposed as an initial product of the thermal degradation of levoglucosan ${ }^{96,97}$ and levogalactosan. ${ }^{45}$ Thus, while the formation of 3,6-Anhyro-Dgalactose was not observed during the simulation, the hydrogen bond between 06 and 03 which endures throughout the simulation of the lowest energy conformer of III suggests that this conformer precedes the formation of neutral 3,6-Anhyro-D-galactose. It is the presence of a strong hydrogen bond between 06 and $\mathrm{O} 3$ in the

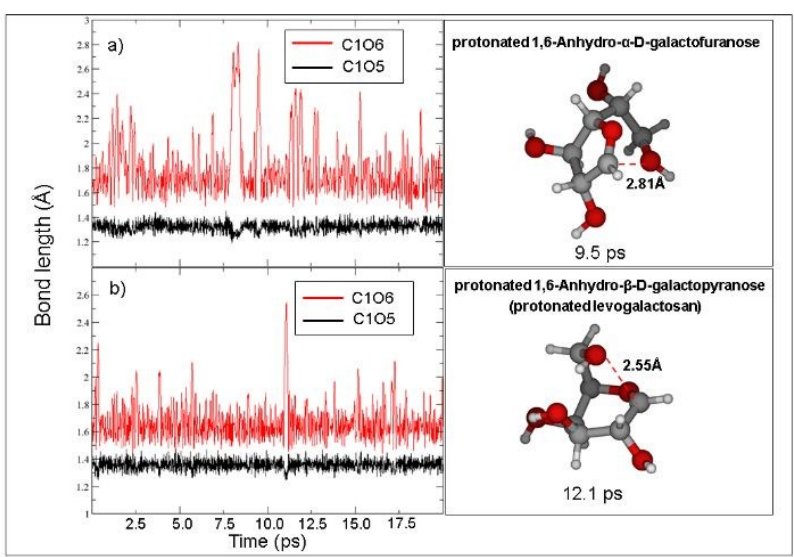

Fig. 9 The evolution of bond distances $\mathrm{C} 106$ (in red) and C1O5 (in black) on a) II and b) III, respectively. Snapshots at maxima of the $\mathrm{C} 106$ bond are shown. lowest energy conformer of III which confers the ion with a spectrial

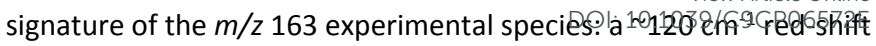
of one of the $\mathrm{OH}$ stretches in the near-IR region (see Figs. 4 and 7). In this way, the IRMPD spectra suggest that the presence of II under the experimental conditions would be minor at best as this $\mathrm{m} / \mathrm{z} 163$ species lacks a strong hydrogen bond that is necessary to account for the red-shifted $\mathrm{OH}$ stretch at $\sim 3750 \mathrm{~cm}^{-1}$. Thus, while energetics and calculated spectra point to the presence of protonated levogalactosan among the $m / z 163$ experimental species, there was no indication that protonated 1,6 -Anhydro- $\alpha$-Dgalactofuranose had been formed from protonated methyl Dgalactopyranoside generated through ESI in the gas phase.

\section{Conclusions}

IRMPD spectra of protonated $\alpha$ - and $\beta$-anomers of methyl D-galactopyranoside and a $m / z 163$ fragment were compared with calculated spectra based on AIMD simulations at temperatures of $\sim 350 \mathrm{~K}$ and VSCF calculations for low energy conformers of protonated methyl D-galactopyranoside, galactosyl oxocarbenium ions, and protonated anhydrogalactoses. Conformational changes in the course of the dynamics were explored and characterized. These findings demonstrate the occurrence of conformational changes on an ultrafast timescale in protonated sugar species. These transitions must be considered in analysing the spectroscopy of the species. Specific conclusions drawn from the analysis of the spectra and the dynamics of these species include the following:

(1) Protonation of $\alpha$ - and $\beta$ - anomers of methyl Dgalactopyranoside under the experimental conditions leads to a $m / z 195$ species that has a shared proton between $\mathrm{O} 4$ and the hydroxymethyl oxygen which forms an intramolecular proton shared dimer, a motif which may impact the reactivity of this species to acid catalysis. Simulation of the lowest energy conformer of $\mathrm{BMeGal}^{-\mathrm{H}^{+}}$revealed that the out-of-plane ring stabilizes the protonated galactopyranoside, suggesting that the hydroxymethyl position is the preferred site of protonation in the gas phase.

(2) A key finding of theory which seems compatible with experiment is that the lifetimes of conformers of protonated sugars can be of very short magnitude. In these systems the interplay between stability and short range transitions was found to be predominant. Transitions between all five of the galactosyl oxocarbenium ion conformers identified, as well as a 1,4 -Anhydro- $\beta$-D-galactopyranose, were observed during simulations of these species. While formation and breakdown of 1,4-Anhydro- $\beta$-D-galactopyranose was quite rare, the lifetimes of the galactosyl oxocarbenium ion conformers did not exceed 8 ps. Thus, rapid conformer transitions on the picosecond timescale are characteristic of the galactosyl oxocarbenium ion at room temperature. In contrast, simulation of protonated1,6-Anhydro- $\alpha-D$ galactofuranose and protonated levogalactosan revealed these anhydrogalactose cations to be stable 
conformers at $\sim 350 \mathrm{~K}$. However, short femtosecond shifts to an oxocarbenium ion structure were also observed for these species, demonstrating anhydrogalactose stabilization of galactosyl oxocarbenium ions in the gas phase.

(3) Calculated IR spectra from AIMD simulations of galactosyl oxocarbenium ions and protonated anhydrogalactoses suggest that the structure associated with $\mathrm{m} / \mathrm{z} 163$ species is the lowest energy conformer of the galactosyl oxocarbenium ion. However, VSCF calculations of IR spectra of protonated levogalactosan, the lowest energy $\mathrm{m} / \mathrm{z}$ 163 cation identified in this study, are also in good agreement with the IRMPD spectra of the $\mathrm{m} / \mathrm{z} 163$ species. It is not possible to determine its identity unequivocally: either or both of the galactosyl oxocarbenium ion and protonated levogalactosan species may exist under the experimental conditions but as dynamics simulations showed the transient oxocarbenium ion nature of protonated levogalactosan, the presence of an oxocarbenium ion species under the experimental conditions is strongly suggested.

(4) Both classical dynamics and static quantum mechanical VSCF calculations were used to probe the galactosyl oxocarbenium ion and protonated anhydrogalactose systems. This study shows the merits of using this combined approach in analysing the spectra of floppy systems as those under consideration here. For species of short lifetimes such as the highly transient oxocarbenium ion conformers VSCF is problematic. For stable species of welldefined structure such as the levogalactosan cation, the results of VSCF may be better. But for floppy systems at high temperature, where the lifetime of a given conformer may be too short to expect significant contribution to the experimental spectrum, AIMD simulation must be used to determine the suitability of a single structure method like VSCF. Finally, when the presence of multiple species is possible, calculation of spectra by the AIMD method alone may mask the presence of species with less intense spectral features.

\section{Conflicts of interest}

There are no conflicts to declare.

\section{Acknowledgements}

MD thanks Huan Wang for helpful discussions regarding AIMD simulations.

\section{Notes and references}

₹ Conformational effects refer to both those effects relating to whether a substituent is in an equatorial oraxia!1Bositionpard/te torsional changes to the structure that do not involve the inversion of a stereocenter.

1. T. J. Boltje, T. Buskas and G. J. Boons, Nat. Chem., 2009, 1, 611-622.

2. S. S. Nigudkar and A. V. Demchenko, Chem. Sci., 2015, 6, 2687-2704.

3. X. M. Zhu and R. R. Schmidt, Angew. Chem. Int. Edit., 2009, 48, 1900-1934.

4. S. U. Hansen, G. J. Miller, M. J. Cliff, G. C. Jaysonc and J. M. Gardiner, Chem Sci., 2015, 6, 6158-6164

5. M. Islam, G. P. Shinde and S. Hotha, Chem. Sci., 2017, 8, 2033-2038.

6. Y. Wu, D. C. Xiong, S. C. Chen, Y. S. Wang and X. S. Ye, Nat. Commun., 2017, 8, 14851.

A. A. Rosatella, S. P. Simeonov, R. F. M. Frade and C. A. M. Afonso, Green Chem., 2011, 13, 754-793.

12. S. van der Vorm, T. Hansen, H. S. Overkleeft, G. A. van der Marel and J. D. C. Codee, Chem. Sci., 2017, 8, 1867-1875.

13. A. Martin, A. Arda, J. Desire, A. Martin-Mingot, N. Probst, P. Sinay, J. Jimenez-Barbero, S. Thibaudeau and Y. Bleriot, Nat. Chem., 2016, 8, 186191.

14. L. Bohe and D. Crich, Nat. Chem., 2016, 8, 97-99.

15.

C. Denekamp and Y. Sandlers, J. Mass Spectrom., 2005, 40, 765-771. X. C. Huang, C. Surry, T. Hiebert and A. J. Bennet, J. Am. Chem. Soc., 1995, 117, 10614-10621.

P. K. Kancharla, C. Navuluri and D. Crich, Angew. Chem. Int. Edit., 2012, 51 11105-11109.

M. L. Sinnott and W. P. Jencks, J. Am. Chem. Soc., 1980, 102, 2026-2032. J. T. Edward, Chem. Ind. (London), 1955, 36, 1102-1104.

M. Miljkovic, D. Yeagley, P. Deslongchamps and Y. L. Dory, J. Org. Chem., 1997, 62, 7597-7604.

W.G. Overend, C.W. Rees and J.S. Sequeira J. Chem. Soc., 1962, 3429-3440. D. Crich, M. de la Mora and A. U. Vinod, J. Org. Chem., 2003, 68, 8142-8148. Z. Y. Zhang, I. R. Ollmann, X. S. Ye, R. Wischnat, T. Baasov and C. H. Wong, J. Am. Chem. Soc., 1999, 121, 734-753.

H. Elferink, M. E. Severijnen, J. Martens, R. A. Mensink, G. Berden, J. Oomens, F. P. J. T. Rutjes, A. M. Rijs and T. J. Boltje, J. Am. Chem. Soc., 2018, 140, 6034-6038.

S. Rudic, H. B. Xie, R. B. Gerber and J. P. Simons, Mol. Phys., 2012, 110, 16091615.

28. R. Sagar, S. Rudic, D. P. Gamblin, E. M. Scanlan, T. D. Vaden, B. Odell, T. D. W. Claridge, J. P. Simons and B. G. Davis, Chem. Sci., 2012, 3, 2307-2313.

29. E. Mucha, A. I. G. Florez, M. Marianski, D. A. Thomas, W. Hoffmann, W. B. Struwe, H. S. Hahm, S. Gewinner, W. Schollkopf, P. H. Seeberger, G. von Helden and K. Pagel, Angew. Chem. Int. Edit., 2017, 56, 11248-11251.

E. Mucha, M. Lettow, M. Marianski, D. A. Thomas, W. B. Struwe, D. J. Harvey, G. Meijer, P. H. Seeberger, G. von Helden and K. Pagel, Angew. Chem. Int. Edit., 2018, 57, 7440-7443.

31.

E. Mucha, M. Marianski, F. F. Xu, D. A. Thomas, G. Meijer, G. von Helden, P. H. Seeberger and K. Pagel, Nat. Commun., 2018, 9, 4174.

32. E. Mucha, A. Stuckmann, M. Marianski, W. B. Struwe, G. Meijer and K. Pagel, Chem. Sci. 2019, 10, 1272-1284.

33. D. Marx and J. Hutter, Ab initio Molecular Dynamics: Basic Theory and Advanced Methods, Cambridge University Press, Cambridge ; New York, 2009.

M. P. Gaigeot, Phys. Chem. Chem. Phys., 2010, 12, 3336-3359.

L. Jin and R. B. Gerber, Phys. Chem. Chem. Phys., 2012, 14, 13522-13526.

S. S. Lee, S. Park, J. Y. Kim, H. R. Kim, S. Lee and H. B. Oh, Phys. Chem. Chem Phys., 2014, 16, 8376-8383.

M. Pincu, B. Brauer and R. B. Gerber, Phys. Chem. Chem. Phys., 2013, 15, 15382-15391.

M. Pincu and R. B. Gerber, Chem. Phys. Lett., 2012, 531, 52-58.

H. B. Xie and R. B. Gerber, Phys. Chem. Chem. Phys., 2012, 14, 12086-12089. H. B. Xie, L. Jin, S. Rudic, J. P. Simons and R. B. Gerber, J. Phys. Chem. B, 2012, 116, 4851-4859.

41. J. M. Bowman, J Chem. Phys., 1978, 68, 608-610.
42. R. B. Gerber and M. A. Ratner, Chem. Phys. Lett., 1979, 68, 195-198.

43. T. K. Roy and R. B. Gerber, Phys. Chem. Chem. Phys.., 2013, 15, 9468-9492.

44. N. K. Richtmyer, Arch. Biochem. Biophys., 1958, 78, 376-385.

D. Gardiner, J. Chem. Soc. C, 1966, 1473-1476

A. D. Becke, J. Chem. Phys., 1993, 98, 5648-5652. 
47.

48.

49.

50.

51.

52.
53.

54.

55.

56.

57.

58.

59.

60.

61.

62.

63.

64.

65.

66.

67.

68.

69.

70.

71.

72.

73.

74.

75.

76.

78.

79.

80.

81.

82.

83.

84.

85.

86.

87.

88.

89.

90.

91.

92.

93.

94.

95.

96.
C. T. Lee, W. T. Yang and R. G. Parr, Phys. Rev. B, 1988, 37, 785-789.

R. Ahlrichs, M. Bar, M. Haser, H. Horn and C. Kolmel, Chem. Phys. Lett., 1989, 162, 165-169.

T. K. Roy, V. Kopysov, N. S. Nagornova, T. R. Rizzo, O. V. Boyarkin and R. B. Gerber, Chemphyschem, 2015, 16, 1374-1378.

B. Brauer, R. B. Gerber, M. Kabelac, P. Hobza, J. M. Bakker, A. G. A. Riziq and M. S. de Vries, J. Phys. Chem. A, 2005, 109, 6974-6984.

A. Barducci, G. Bussi and M. Parrinello, Phys. Rev. Lett., 2008, 100, 020603. E. K. Peter, I. V. Pivkin and J. E. Shea, J. Chem. Phys., 2016, 145, 044903.

J. VandeVondele, M. Krack, F. Mohamed, M. Parrinello, T. Chassaing and J. Hutter, Comput. Phys. Commun., 2005, 167, 103-128.

P. Hohenberg and W. Kohn, Phys. Rev., 1964, 136, B864.

W. Kohn and L. J. Sham, Phys. Rev., 1965, 140, A1133.

G. M. Chaban and R. B. Gerber, Theor. Chem. Acc., 2008, 120, 273-279.

C. A. Jimenez-Hoyos, B. G. Janesko and G. E. Scuseria, Phys. Chem. Chem. Phys., 2008, 10, 6621-6629.

J. Sebek, R. Knaanie, B. Albee, E. O. Potma and R. B. Gerber, J. Phys. Chem. A, 2013, 117, 7442-7452.

M. P. Gaigeot, R. Vuilleumier, M. Sprik and D. Borgis, J. Chem. Theory Comput., 2005, 1, 772-789.

S. Goedecker, M. Teter and J. Hutter, Phys. Rev. B, 1996, 54, 1703-1710.

G. J. Martyna and M. E. Tuckerman, J. Chem. Phys., 1999, 110, 2810-2821.

W. G. Hoover, Phys. Rev. A, 1985, 31, 1695-1697.

S. Nose, J. Chem. Phys., 1984, 81, 511-519.

D. A. McQuarrie, Statistical Mechanics, University Science Books, Sausalito, Calif., 2000.

A. Khintchine, Math. Ann., 1934, 109, 604.

N. Wiener, Acta Math., 1930, 55

M. Frigo and S. G. Johnson, P. leee, 2005, 93, 216-231.

M. Bernasconi, P. L. Silvestrelli and M. Parrinello, Phys. Rev. Lett., 1998, 81, 1235-1238.

J. M. Bowman, Accounts Chem. Res., 1986, 19, 202-208.

R. B. Gerber and A. Amirav, J. Phys. Chem., 1986, 90, 4483-4491.

R. B. Gerber and M. A. Ratner, Adv. Chem. Phys., 1988, 70, 97-132.

J. O. Jung and R. B. Gerber, J. Chem. Phys., 1996, 105, 10332.

L. Pele, J. Sebek, E. O. Potma and R. B. Gerber, Chem. Phys. Lett., 2011, 515,

7-12.

J. Sebek, L. Pele, E. O. Potma and R. B. Gerber, Phys. Chem. Chem. Phys., 2011, 13, 12724-12733.

L. S. Norris, M. A. Ratner, A. E. Roitberg and R. B. Gerber, J. Chem. Phys., 1996, 105, 11261.

T. K. Roy, R. Sharma and R. B. Gerber, Phys. Chem. Chem. Phys., 2016, 18, 1607-1614.

M. W. Schmidt, K. K. Baldridge, J. A. Boatz, S. T. Elbert, M. S. Gordon, J. H. Jensen, S. Koseki, N. Matsunaga, K. A. Nguyen, S. J. Su, T. L. Windus, M. Dupuis and J. A. Montgomery, J. Comput. Chem., 1993, 14, 1347-1363.

J. M. Bakker, T. Besson, J. Lemaire, D. Scuderi and P. Maitre, J. Phys. Chem. A, 2007, 111, 13415-13424.

R. Prazeres, F. Glotin, C. Insa, D. A. Jaroszynski and J. M. Ortega, Eur. Phys. J. D, 1998, 3, 87-93.

J. M. Bakker, R. K. Sinha, T. Besson, M. Brugnara, P. Tosi, J. Y. Salpin and P. Maitre, J. Phys. Chem. A, 2008, 112, 12393-12400.

R. K. Sinha, E. Nicol, V. Steinmetz and P. Maitre, J. Am. Soc. Mass Spectrom., 2010, 21, 758-772.

C. S. Contreras, N. C. Polfer, J. Oomens, J. D. Steill, B. Bendiak and J. R. Eyler, Int. J. Mass Spectrom., 2012, 330, 285-294.

C. Fraschetti, L. Guarcini, C. Zazza, L. Mannina, S. Circi, S. Piccirillo, B. Chiavarino and A. Filippi, Phys. Chem. Chem Phys., 2018, 20, 8737-8743.

J. R. Roscioli, L. R. McCunn and M. A. Johnson, Science, 2007, 316, 249-254.

P. Ecija, I. Uriarte, L. Spada, B. G. Davis, W. Caminati, F. J. Basterretxea, A. Lesarri and E. J. Cocinero, Chem. Commun., 2016, 52, 6241-6244.

D. Cremer and J. A. Pople, J. Am. Chem. Soc., 1975, 97, 1354-1358. I. Tvaroska, F. R. Taravel, J. P. Utille and J. P. Carver, Carbohyd. Res., 2002, 337, 353-367.

E. J. Cocinero, A. Lesarri, P. Ecija, F. J. Basterretxea, J. U. Grabow, J. A. Fernandez and F. Castano, Angew. Chem. Int. Edit., 2012, 51, 3119-3124.

T. Nukada, A. Berces, L. J. Wang, M. Z. Zgierski and D. M. Whitfield, Carbohyd Res., 2005, 340, 841-852.

P. L. Polavarapu and C. S. Ewig, J. Comput. Chem., 1992, 13, 1255-1261.

E. J. Cocinero, A. Lesarri, P. Ecija, A. Cimas, B. G. Davis, F. J. Basterretxea, J. A. Fernandez and F. Castano, J. Am. Chem. Soc., 2013, 135, 2845-2852. G. M. Chaban, R. B. Gerber and K. C. Janda, J. Phys. Chem. A, 2001, 105, 8323-8332.

G. M. Chaban, J. O. Jung and R. B. Gerber, J. Phys. Chem. A, 2000, 104, 27722779.

G. M. Chaban, S. S. Xantheas and R. B. Gerber, J. Phys. Chem. A, 2003, 107 4952-4956.

Y. Miller, G. M. Chaban and R. B. Gerber, Chem. Phys., 2005, 313, 213-224. M. R. Nimlos and R. J. Evans, Fuel Chem. Division Preprints, 2002, 47, 393 394. 